\title{
A simple extended compact nonlinear scheme with adaptive dissipation control
}

\author{
Huaibao Zhang ${ }^{\mathrm{a}}$, Fan Zhang ${ }^{\mathrm{b}}$, Jun Liu ${ }^{\mathrm{c}}$, J. M. McDonough ${ }^{\mathrm{d}}$, Chunguang Xu $\mathrm{u}^{\mathrm{e}, *}$ \\ ${ }^{a}$ School of Physics, Sun Yat-sen University, Guangzhou 510006, China \\ ${ }^{b}$ Centre for mathematical Plasma-Astrophysics, Department of Mathematics, KU Leuven, Celestijnenlaan $200 B, 3001$ \\ Heverlee, Belgium \\ ${ }^{c}$ School of Aeronautics and Astronautics, Dalian University of Technology, Dalian 116024, China \\ ${ }^{d}$ Departments of Mechanical Engineering and Mathematics, University of Kentucky, Lexington, KY 40506, USA \\ ${ }^{e}$ School of Aeronautics and Astronautics, Sun Yat-sen University, Guangzhou 510006, China
}

\begin{abstract}
Weighted compact nonlinear schemes (WCNSs) [1] are a popular family of high resolution shock-capturing methods, of which the nonlinear interpolation procedure is essential for non-oscillatory shock-capturing computation. In this article, numerical dissipation of an improved compact nonlinear scheme, which is fifthorder accurate, is significantly reduced by further introducing a six-point optimized stencil in the nonlinear interpolation, without using more complex local or global smoothness indicators. Nonlinear dissipation and dispersion are then further reduced by using an adaptive strategy. In particular, the computational procedure including the proposed six-point nonlinear interpolation is almost the same as that of the improved five-point scheme, and thus their computational efforts are similar. However, significant gains in numerical performance are obtained while including the six-point optimized stencil. Benchmark cases in one and two space dimensions are used to assess performance of the present scheme, viz., shock-capturing capability and resolution in smooth regions, showing promising advantages.
\end{abstract}

Keywords: compact nonlinear schemes; nonlinear interpolation; high-order schemes; extended stencil

\section{Introduction}

Accurate simulations of compressible flows involving discontinuities such as shocks and interfaces, as well as small-scale flow structures, have received considerable attention in the computational fluid dynamics (CFD) research community over the past decades. Advanced high-order CFD schemes are capable of capturing discontinuities with high resolution, and simultaneously resolving complicated flow structures, as introduced by numerous publications [2, 3, 4, 5. High-order numerical schemes have undergone extensive development, and this is still an active topic with much yet to be done as noted by Wang et al. [6].

Among high-order shock-capturing methods, a popular family of schemes is the weighted compact nonlinear schemes (WCNSs) developed by Deng and Zhang [1]. WCNSs are extension of the compact nonlinear

\footnotetext{
* Corresponding authors.

Email addresses: zhanghb28@sysu.edu.cn (Huaibao Zhang), fan.zhang@kuleuven.be (Fan Zhang), liujun65@dlut.edu.cn (Jun Liu), jmmcd@uky.edu ( J. M. McDonough), xuchg5@sysu.edu.cn (Chunguang Xu)
} 
scheme (CNS) of Deng and Maekawa [7, which provides excellent resolution for high-frequency waves due to the spectral-like resolution of compact schemes proposed by Lele [8], among others. As shown by Nonomura et al. 9, 10, Deng et al. [11, and Wong and Lele [12, WCNSs have several advantages over the classical finite-difference WENO scheme of Jiang and Shu [13, including: (1) resolution is slightly higher; (2) choice of numerical flux schemes is flexible [14; and (3) WCNSs perform well with respect to freestream and vortex preservation properties on wavy grids. Besides these advantages of solving general gas dynamic problems, WCNSs are also more effective to achieve pressure equilibrium while simulating multi-component flows [15].

The classical WCNS procedure consists of three steps [1: (1) node-to-midpoint weighted nonlinear interpolation of flow variables, (2) evaluation of fluxes at midpoints, and (3) midpoint-to-node centered flux differencing. In the first step the idea of nonlinear weighting, originating from WENO schemes [16], is used. The classical WCNS using the JS weight of Jiang and Shu [13] is therefore referred to as WCNS-JS. It has been demonstrated that the nonlinear interpolation procedure is essential for shock capturing ability and resolution of WCNSs [17, 18, 19. Hence, improving this procedure has been attracting much attention.

Recently, focusing on improving the performance of the nonlinear interpolation, the ENO-like stencil selection used by TENO schemes [20] was incorporated in WCNS, resulting in a new compact nonlinear scheme [21, exhibiting reduced numerical dissipation and dispersion. In 21], flux differencing in the third step is performed using an explicit scheme, as used in the WCNS-E-5 [22]. In fact, as suggested by Deng et al. 23], for a WCNS, the nonlinear interpolation in step (1) dominates the resolution property, and explicit centered differencing, which does not need tridiagonal inversion for derivative calculations, is favored due to simplicity of implementation and superior computational efficiency. Further work of Nonomura et al. 24] demonstrated that the type of flux differencing employed does not significantly change resolution, even for higher-order WCNSs. Therefore, in the present work, only the nonlinear interpolation is investigated. It should be noted that, first order interpolation was recommended if the interpolated density or pressure becomes locally negative [12, thus improving the robustness of traditional WCNSs. In this work, this treatment is avoided, and robustness will be further investigated separately.

The effort of this work, again, explores development of low dissipation and dispersion compact nonlinear schemes. Inspired by the work of Zhang et al. [25, 26], we propose to implement a six-point optimized stencil without using more complex smoothness measurement, or storing extra smoothness information, prior to nonlinear interpolation. Moreover, realizing that nonlinear dissipation is controllable via the ENO-like stencil selection procedure, which acts as a discontinuity-detector 27, we apply an adaptive strategy for controlling numerical dissipation and achieving the optimal spectral property.

For introducing the ideas of this work, the remainder of this article is organized as follows. In the next section, the fundamentals and basic equations of compact nonlinear schemes are introduced, and the proposed schemes are derived in Section 3 , which also includes discussion of the approximate dispersion relation (ADR) analysis [28. Section 4 presents solutions of scalar equations and the Euler equations, and concluding remarks are given in the last section. 


\section{Fundamentals}

The governing equations of compressible flow are hyperbolic systems. Without loss of generality, the one-dimensional scalar hyperbolic conservation law is used for discussing the numerical schemes involved in this work, and takes the form

$$
\frac{\partial u}{\partial t}+\frac{\partial f(u)}{\partial x}=0
$$

subject to the initial condition

$$
u(x, 0)=u_{0}(x),
$$

where $u$ is the dependent variable, and $f(u)$ is the flux term. The governing equation (1), together with its initial condition (2), composes an initial-value problem (IVP), which is assumed to be well-posed in the sense that its exact solution $u$ continuously depends on the initial field and is piecewise smooth with a finite number of discontinuities.

Spatial discretization of Eq. (1) is performed on an equally-spaced one-dimensional grid, and the distance between two adjacent grid nodes is denoted as $h$. At each node $i, x_{i}=i h$, and $u_{i}=u\left(x_{i}, t\right)$. The characteristic velocity in Eq. (1) is $\partial f(u) / \partial u$, which is assumed to be positive, without loss of generality. Therefore, at each node, a semi-discrete solution can be constructed:

$$
\left(\frac{\partial u}{\partial t}\right)_{i}=-\hat{f}_{i}^{\prime}, \quad i=1, \cdots, n
$$

where $\hat{f}_{i}{ }^{\prime}$ is the approximation to the first spatial derivative of the numerical flux function $f_{i}$.

In WCNSs, $\hat{f}_{i}^{\prime}$ can be approximated using a centered compact scheme 22, which is written in a general form as

$$
\kappa \hat{f}_{i-1}^{\prime}+\hat{f}_{i}^{\prime}+\kappa \hat{f}_{i+1}^{\prime}=\frac{a_{1}}{h}\left(\widetilde{f}_{i+\frac{1}{2}}-\widetilde{f}_{i-\frac{1}{2}}\right)+\frac{a_{2}}{h}\left(\widetilde{f}_{i+\frac{3}{2}}-\widetilde{f}_{i-\frac{3}{2}}\right)+\frac{a_{3}}{h}\left(\widetilde{f}_{i+\frac{5}{2}}-\widetilde{f}_{i-\frac{5}{2}}\right),
$$

the solution of which involves tridiagonal inversions if $\kappa \neq 0$. As mentioned in the Introduction, an explicit formula is preferred due to its lower computational cost, and thus $\kappa=0$ is set for the following discussions. The sixth-order explicit formula given in [10], termed midpoint-and-node-to-node difference (MND), is specifically applied in this work. The formula is

$$
\hat{f}_{i}^{\prime}=\frac{b_{1}}{h}\left(\hat{f}_{i+\frac{1}{2}}-\hat{f}_{i-\frac{1}{2}}\right)+\frac{b_{2}}{h}\left(f_{i+1}-f_{i-1}\right)+\frac{b_{3}}{h}\left(\hat{f}_{i+\frac{3}{2}}-\hat{f}_{i-\frac{3}{2}}\right),
$$

where the coefficients have values $b_{1}=3 / 2, b_{2}=-3 / 10$, and $b_{3}=1 / 30$.

Apparently, this explicit differencing scheme is not strictly a compact scheme but is considered a special case of general compact nonlinear schemes. Moreover, the present work focuses on nonlinear interpolation of flow variables, without concern for the centered-differencing procedure employed. The present method can also be implemented with using the implicit formula in Eq. (4).

Midpoint flux terms in Eq. (5) are unknown and can be evaluated using numerical upwind flux functions. The scalar upwind flux function is given in generic form as

$$
\hat{f}_{i \pm \frac{1}{2}}=\frac{1}{2}\left[\left(f\left(u_{R, i \pm \frac{1}{2}}\right)+f\left(u_{L, i \pm \frac{1}{2}}\right)\right)-|\hat{a}|\left(u_{R, i \pm \frac{1}{2}}-u_{L, i \pm \frac{1}{2}}\right)\right],
$$


where the subscripts, viz., $L$ and $R$, indicate, respectively, the variables on the left- and right-hand sides of the midpoint $x_{i \pm \frac{1}{2}}$, and $\hat{a}$ is the approximate eigenvalue of the flux Jacobian matrix. The method for calculating the midpoint variables $u_{L / R, i \pm \frac{1}{2}}$ is described in the next section.

Once the numerical approximation $\hat{f}_{i}^{\prime}$ is given, temporal integration of Eq. (3) is performed using the third-order strongly stable Runge-Kutta method [29].

\section{The present method}

As mentioned above, in order to calculate $u_{L / R, i \pm \frac{1}{2}}$, we had proposed an improved compact nonlinear scheme 21] using the ENO-like stencil selection procedure [20], showing low dissipation and dispersion, in the nonlinear interpolation procedure. A further improvement focusing on nonlinear interpolation is investigated in the current work so that the overall performance of this compact nonlinear scheme can be enhanced accordingly. For simplicity, we only consider the evaluation of variables on the left- and right-hand sides of the midpoint $x_{i+\frac{1}{2}}$, here denoted as $u_{L / R, i+\frac{1}{2}}$.

\subsection{The smoothness measurement}

Before performing nonlinear interpolations, locations of discontinuities within the range of the candidate stencils must be determined. In order to measure the smoothness of flow field variables, local and global smoothness indicators are constructed.

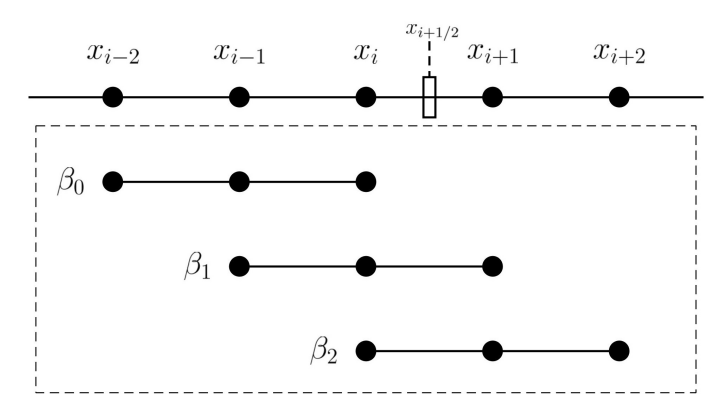

Figure 1: Local smoothness indicators.

As shown in Fig. 1. evaluation of smoothness measurement is applied on a five-point full stencil $\mathrm{SM}_{L, i+\frac{1}{2}}=$ $\left\{x_{i-2}, x_{i-1}, x_{i}, x_{i+1}, x_{i+2}\right\}$, where SM denotes "the stencil used for smoothness measurement". Just as in WENO schemes [16, 13], three three-point substencils with index $k$, namely,

$$
\mathrm{SM}_{L, i+\frac{1}{2}, k}=\left\{x_{i+k-2}, x_{i+k-1}, x_{i+k}\right\}, \quad k=0,1,2
$$

are used to compute three local smoothness indicators, the $\beta_{k}$ s. Considering that each of the third-order interpolations evaluated on its corresponding substencil can be expressed in a generic form using (approximated) $n^{\text {th }}$ derivatives $(n=1,2)$, a local Taylor polynomial can be constructed

$$
u_{L, i+\frac{1}{2}, k}=u\left(x_{i}+\Delta x\right)=u_{i}+u_{i, k}^{(1)} \Delta x+u_{i, k}^{(2)} \frac{\Delta x^{2}}{2},
$$


where $\Delta x=x_{i+\frac{1}{2}}-x_{i}=h / 2$, with first- and second-order differences, given by

$$
\begin{aligned}
& u_{i, 0}^{(1)}=\frac{1}{2 h}\left(u_{i-2}-4 u_{i-1}+3 u_{i}\right), \\
& u_{i, 1}^{(1)}=\frac{1}{2 h}\left(u_{i+1}-u_{i-1}\right), \\
& u_{i, 2}^{(1)}=\frac{1}{2 h}\left(-3 u_{i}+4 u_{i+1}-u_{i+2}\right),
\end{aligned}
$$

and

$$
\begin{aligned}
u_{i, 0}^{(2)} & =\frac{1}{h^{2}}\left(u_{i-2}-2 u_{i-1}+u_{i}\right), \\
u_{i, 1}^{(2)} & =\frac{1}{h^{2}}\left(u_{i-1}-2 u_{i}+u_{i+1}\right), \\
u_{i, 2}^{(2)} & =\frac{1}{h^{2}}\left(u_{i}-2 u_{i+1}+u_{i+2}\right),
\end{aligned}
$$

respectively. Then $\beta_{k}$ is defined as [1]

$$
\beta_{k}=\left(h u_{i, k}^{(1)}\right)^{2}+\left(h^{2} u_{i, k}^{(2)}\right)^{2}
$$

Following the procedure in [21], the smoothness measurement for the $k^{t h}$ candidate substencil is calculated as

$$
\gamma_{k}=\left(C+\frac{\tau_{5}}{\beta_{k}+\epsilon}\right)^{q}, \quad k=0,1,2,
$$

where the global smoothness indicator is defined as $\tau_{5}=\left|\beta_{0}-\beta_{2}\right|$. The small threshold value employed for the reported studies was $\epsilon=10^{-12}$, which is consistent with the double precision arithmetic used here. The two constant parameters resulting in strong scale separation have values $C=1$ and $q=6$ [20]. Instead of using the original nonlinear smoothness measurement in Eq. [12], $\gamma_{k}$ is further normalized as

$$
\chi_{k}=\frac{\gamma_{k}}{\sum_{k=0}^{2} \gamma_{k}} .
$$

Then a sharp cut-off function can be defined as

$$
\delta_{k}=\left\{\begin{array}{lc}
0, & \text { if } \quad \chi_{k}<C_{T} \\
1, & \text { otherwise }
\end{array}\right.
$$

where $C_{T}$ serves as the global reference for smoothness indicators, and a constant value $C_{T}=10^{-5}$ was used for computations of several five point schemes [20, 21]. Here, if $\delta_{k}$ equals zero, the polynomial evaluated on the corresponding substencil is deemed to be "oscillatory"; otherwise the substencil is regarded as "smooth". Therefore, by using this procedure, locations of discontinuities within the range of the five-point full stencil can be determined. The smoothness measurement on the right-hand side of $x_{i+\frac{1}{2}}$ can be defined symmetrically, and thus the corresponding discussion is omitted.

\subsection{Adaptive nonlinear dissipation}

It has also been found that appropriately distinguishing discontinuities may fully recover spectral properties of the underlying linear schemes as shown in Deng et al. [30]. In [21], as noted above, we employed a 
constant value of $C_{T}$, but this may not always be optimal. and thus an alternative is the adaptive nonlinear dissipation control [31] which uses a detector $m$, originally from the work of Ren et al. [32, given by

$$
m=1-\min \left(1, \frac{\eta_{i+\frac{1}{2}}}{C_{r}}\right)
$$

to indicate the potential discontinuity location. Here

$$
\eta_{i+\frac{1}{2}}=\min \left(\eta_{i-1}, \eta_{i}, \eta_{i+1}\right), \quad \eta_{i}=\frac{\left|2 \Delta f_{i+\frac{1}{2}} \Delta f_{i-\frac{1}{2}}\right|+\epsilon}{\left(\Delta f_{i+\frac{1}{2}}\right)^{2}+\left(\Delta f_{i-\frac{1}{2}}\right)^{2}+\epsilon}, \quad \text { and } \quad \epsilon=\frac{0.9 C_{r}}{1-0.9 C_{r}} \xi^{2} .
$$

Specifically, the two parameters involved in Eq. 16 take values $C_{r}=0.24$ and $\xi=10^{-3}$. The parameter $C_{T}$ in Eq. 14 can now be adaptively determined using

$$
\begin{aligned}
g(m) & =\left(1-m^{4}\right)(1+4 m), \\
\bar{\beta} & =\alpha_{1}-\alpha_{2}(1-g(m)), \\
C_{T} & =10^{-\lfloor\bar{\beta}\rfloor},
\end{aligned}
$$

80

$$
\text { to }
$$

\section{to} likely to be smooth, further reducing nonlinear numerical dissipation and dispersion; and a large $C_{T}$ which ensures shock-capturing stability will be obtained if there is potentially a discontinuity.

\subsection{The new stencil-selection procedure}

Once locations of discontinuities are determined using the aforementioned procedure of smoothness measurement, high-order interpolations can be constructed on only smooth stencils shown in Fig. 2, labeled $\mathrm{SP}_{l}$ (stencils used for constructing polynomials), without crossing any discontinuities. Note that SMs in the last subsection are only utilized for smoothness measurement, and the traditional concept of "convex combination" [16] is not applied in this work. Instead, the concept of "stencil-selection procedure" is applied for final high-order interpolations, yielding more flexibility in constructing new schemes [27]. The idea of the optimal candidate stencil selection is straightforward: choose the largest smooth stencil. The algorithm for constructing a five-point scheme is shown in Algorithm 1. With using this algorithm and the adaptive nonlinear dissipation for nonlinear interpolations, the corresponding compact nonlinear scheme is denoted as TCNS5-II.

\subsection{Extended nonlinear interpolation: a six-point interpolation}

A six-point stencil $\mathrm{SP}_{6}$ was presented in Fig. 2] This stencil exceeds the range of $\mathrm{SM}_{L, i+\frac{1}{2}}$, and thus cannot be applied based only on the smoothness information of the $\left\{\delta_{k} \mid k=0,1,2\right\}$ on $\mathrm{SM}_{L, i+\frac{1}{2}}$. However, a simple solution for this can be used, as shown in Fig. 3. Two five-point full stencils, which are usually used for the five-point interpolations of $u_{L, i+\frac{1}{2}}$ and $u_{R, i+\frac{1}{2}}$, respectively, cover the whole range of the centered six-point stencil $\mathrm{SP}_{6}$. Therefore, if both five-point full stencils are smooth, a six-point interpolation can 


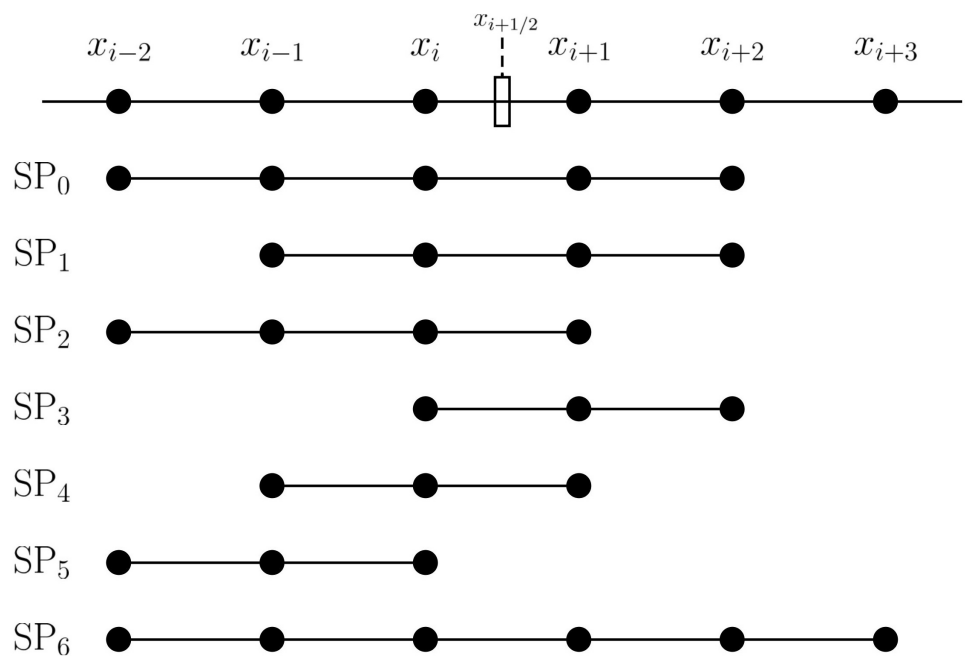

Figure 2: Candidate stencils for high-order interpolations.

be used for calculating $u_{L / R, i+\frac{1}{2}}$. In fact, the code structure can be elegantly designed in such a way that the smoothness information of both five-point full stencils is easily accessible for this purpose. To demonstrate this, we introduce Algorithm 2 below. With using this algorithm for nonlinear interpolations, the corresponding six-point compact nonlinear scheme is denoted as TCNS5-E.

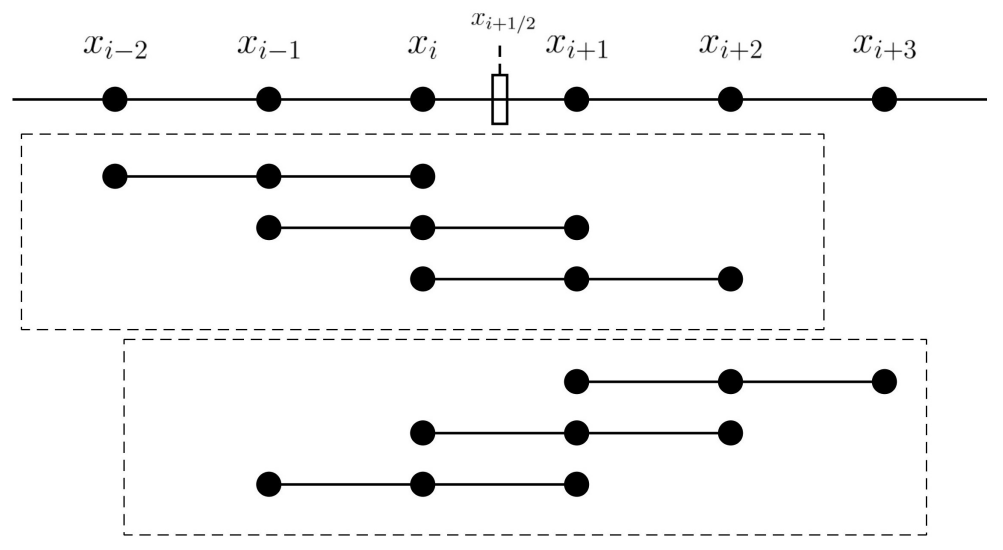

Figure 3: Left and right SMs in the relevant range of $x_{i+\frac{1}{2}}$ for measuring smoothness.

105

\subsection{High-degree polynomials}

Coefficients of the high-degree polynomials $\mathrm{P}_{l, L}=\sum_{m=i-2}^{i+2} a_{l, m} u_{m}$ corresponding to the candidate stencils $\mathrm{SP}_{l}$ are shown in Table 1 for interpolating $u_{L, i+\frac{1}{2}}$. The polynomials for interpolating $u_{R, i+\frac{1}{2}}$ can be given symmetrically, and thus we drop the subscript $L$ for simplicity. For $\mathrm{P}_{0}$ through $\mathrm{P}_{5}$, the polynomials are constructed for achieving the highest-order of accuracy on given stencils, whereas, a different strategy is used to compute the six-point interpolation, i.e., $\mathrm{P}_{6}$.

A sixth-order centered scheme can be defined on $\mathrm{SP}_{6}$, without numerical dissipation. However, numerical 
Table 1: Coefficients of polynomials for high-degree nonlinear interpolation.

\begin{tabular}{ccccccc}
\hline $\mathrm{P}_{l}$ & $a_{l, i-2}$ & $a_{l, i-1}$ & $a_{l, i}$ & $a_{l, i+1}$ & $a_{l, i+2}$ & $a_{l, i+3}$ \\
\hline $\mathrm{P}_{0}$ & $3 / 128$ & $-5 / 32$ & $45 / 64$ & $15 / 32$ & $-5 / 128$ & 0 \\
$\mathrm{P}_{1}$ & 0 & $-1 / 16$ & $9 / 16$ & $9 / 16$ & $-1 / 16$ & 0 \\
$\mathrm{P}_{2}$ & $1 / 16$ & $-5 / 16$ & $15 / 16$ & $5 / 16$ & 0 & 0 \\
$\mathrm{P}_{3}$ & 0 & 0 & $3 / 8$ & $3 / 4$ & $-1 / 8$ & 0 \\
$\mathrm{P}_{4}$ & 0 & $-1 / 8$ & $3 / 4$ & $3 / 8$ & 0 & 0 \\
$\mathrm{P}_{5}$ & $3 / 8$ & $-5 / 4$ & $15 / 8$ & 0 & 0 & 0 \\
$\mathrm{P}_{6}$ & $9 / 640$ & $-7 / 64$ & $39 / 64$ & $9 / 16$ & $-11 / 128$ & $3 / 320$ \\
\hline
\end{tabular}

dissipation in high-wavenumber regions is necessary and beneficial for numerical stability. Following the optimization method given in [20], a six-point interpolation of fifth-order accuracy is constructed as

$$
\mathrm{P}_{6}=\eta \mathrm{p}_{i+\frac{1}{2}, 1}+(1-\eta) \mathrm{p}_{i+\frac{1}{2}, 2}
$$

using two five-point polynomials

$$
\begin{aligned}
& \mathrm{p}_{i+\frac{1}{2}, 1}=\frac{1}{128}\left(3 u_{i-2}-20 u_{i-1}+90 u_{i}+60 u_{i+1}-5 u_{i+2}\right), \\
& \mathrm{p}_{i+\frac{1}{2}, 2}=\frac{1}{128}\left(-5 u_{i-1}+60 u_{i}+90 u_{i+1}-20 u_{i+2}+3 u_{i+3}\right),
\end{aligned}
$$

in which, $\mathrm{p}_{i+\frac{1}{2}, 1}$ is a fifth-order upwind scheme, and $\mathrm{p}_{i+\frac{1}{2}, 2}$ is a fifth-order downwind scheme. If the weight $\eta=0.5$, the sixth-order centered scheme is obtained from Eq. [18), and if $\eta=1.0$, the fifth-order upwind scheme, i.e., $\mathrm{P}_{0}$, is recovered. Therefore, increasing $\eta$ produces a more dissipative upwind scheme, but dissipation is still reduced compared with the fifth-order upwind scheme as long as $\eta<1$, due to the antidissipation property of $\mathrm{p}_{i+\frac{1}{2}, 2}$. In this work, $\eta=0.6$ is applied in most numerical simulations of the following section, while other choices are also applicable. More specific discussions are given in Section 3.6, to explain the effect of the simple strategy designed for the six-point interpolation.

We can now compare the five-point and six-point methods. As mentioned above, Algorithm 1 is provided to explain the procedure of constructing a five-point scheme, where $\mathrm{P}_{l}$ is the high-order polynomial evaluated on candidate stencil $\mathrm{SP}_{l}$. For comparison, another six-point interpolation scheme with using the optimization procedure in Ref. 20] and the adaptive nonlinear dissipation [31] is also introduced in the Appendix.

Remark 1. In a typical WCNS, if only the central substencil, i.e. $\mathrm{SM}_{1}$, is deemed to be oscillatory, the other two stencils are applied in convex combination [1]. However, this combination produces an interpolation which, in fact, still crosses the discontinuity located within the range of $\mathrm{SM}_{1}$. This specific dilemma, however, is avoided in the current method as shown below in Algorithm 1. In particular, if $\mathrm{SM}_{1}$ is oscillatory while $\mathrm{SM}_{0}$ and $\mathrm{SM}_{2}$ are both smooth, $\mathrm{SP}_{3}$ is applied for the interpolation since the midpoint $x_{i+\frac{1}{2}}$ is located within the range of $\mathrm{SP}_{3}$. 


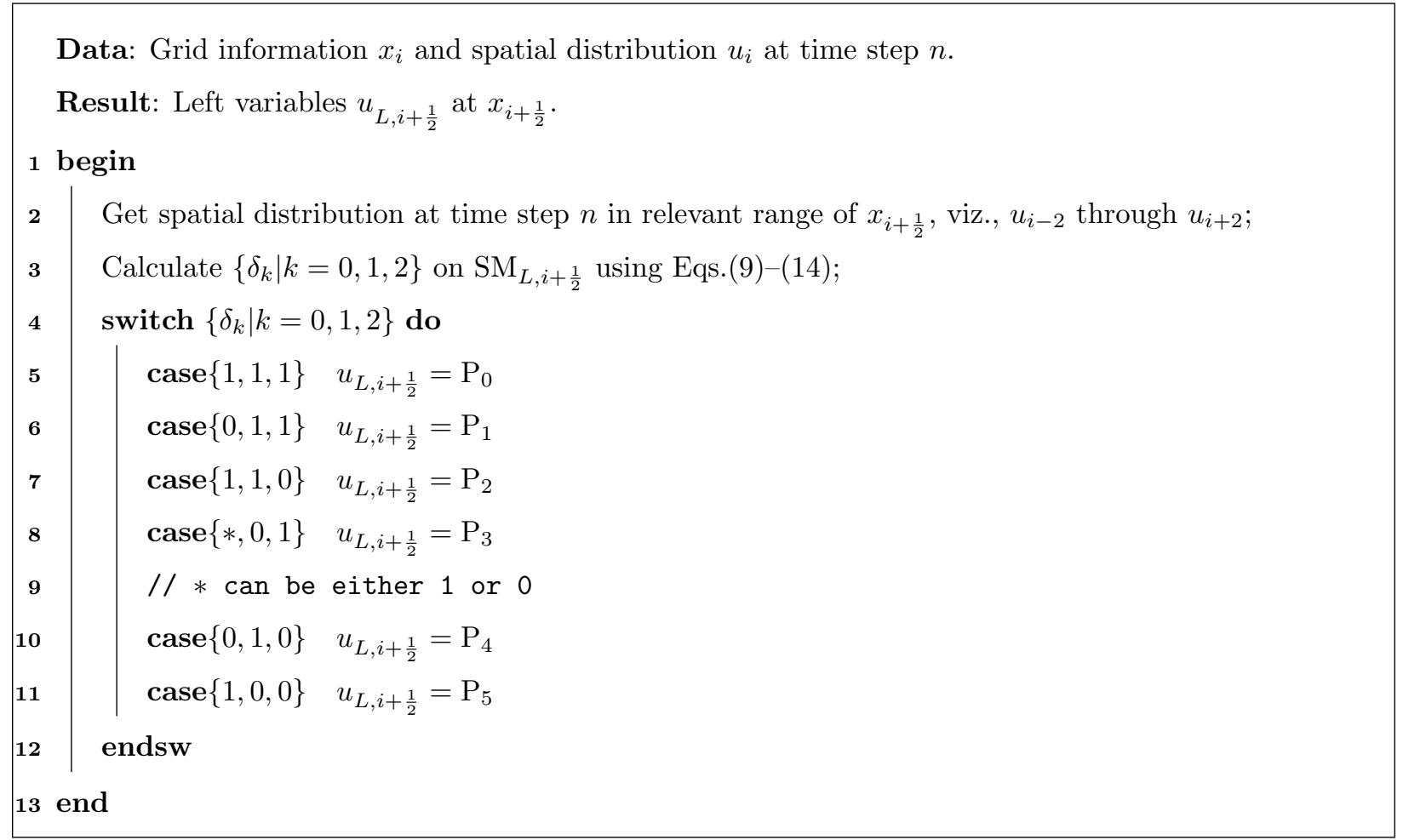

Algorithm 1: Constructing the five-point scheme.

Remark 2. The underlying philosophy of designing the present algorithm is partly inspired by the MOOD paradigm [33, 34, which provides optimal accuracy while satisfying the discrete maximum principle (DMP) cedure as is MOOD, it provides high-order approximation with guaranteeing the ENO property (instead of satisfying DMP), and reduces the order of accuracy if the ENO property might be violated. An a posteriori paradigm achieving oscillation-free interpolations can also be implemented using these SPs, and details will be discussed in future work.

Remark 3. Discarding the concept of "convex combination" [16] allows use of optimal linear schemes on all SPs. Particularly, optimization can be performed for a specific interpolation, e.g., $\mathrm{P}_{0}$, to improve the dissipation and/or dispersion properties, without changing other interpolations. This flexibility results in minor improvement described in this section, while the idea is exploited further in the following sections.

As is shown, the selection procedure in this algorithm (lines 8 through 15) is exactly the same as that in Algorithm 1, except that both left and right interpolations are now considered at the same time. The difference lies in lines 3,4 and 5, where a six-point interpolation can be applied for interpolating the variables on both sides of the midpoint $x_{i+\frac{1}{2}}$ in smooth flow fields.

We are able to use the six-point centered stencil in the nonlinear interpolation by making the most of smoothness measurements already available, and the present interpolation method is thus called an extended nonlinear interpolation procedure. This idea, as mentioned above, is motivated by [25, 26], in which proce- 


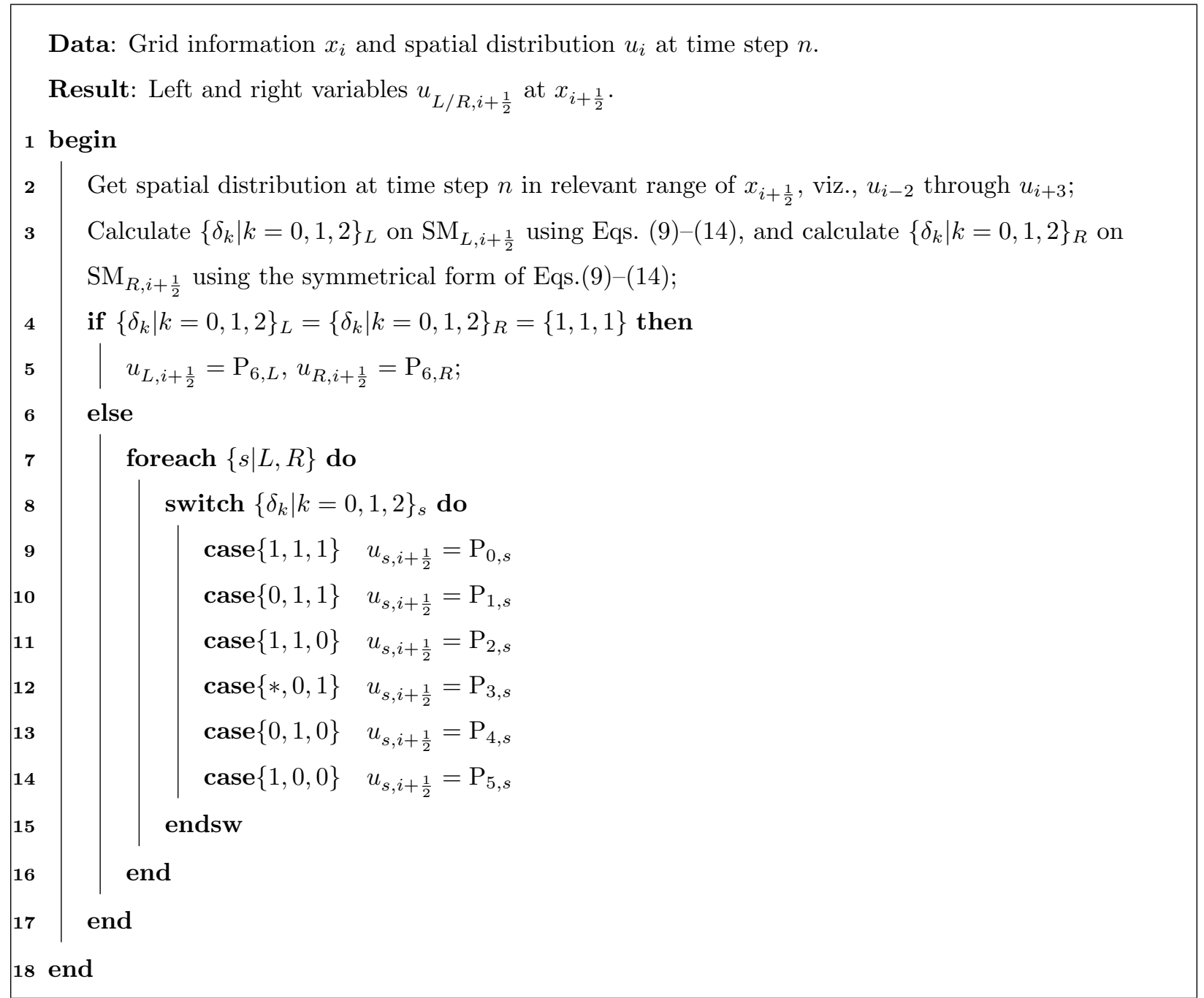

Algorithm 2: Constructing the six-point scheme.

dures of smoothness measurement and high-order interpolation are separated, and smoothness information must be stored before the high-order interpolation is performed. In the present work, however, extra storage of smoothness information is not required, and the algorithm is simpler to implement. In the following discussions, this new six-point scheme is simply denoted as TCNS5-E to emphasize the idea of extending the basic strategy being used.

\subsection{Approximate dispersion relation}

Here, the approximate dispersion relation (ADR) analysis [28] is performed to evaluate spectral properties of the numerical schemes considered in the present work, viz., WCNS-JS, TCNS5, TCNS5-II, TCNS5-E and the TCNS6 introduced in the appendix. Particularly, the ADR analysis shows the dissipation and dispersion errors of nonlinear numerical schemes in wavenumber space by solving a linear advection equation with a smooth initial condition including different Fourier modes supported on a given grid. A modified wavenumber $\xi$ (normalized to lie in the interval $[0, \pi]$ ) is defined for all supported modes, and its real $\left(\xi_{R}\right)$ 
and imaginary $\left(\xi_{I}\right)$ parts represent dispersion and dissipation properties, respectively, for the numerical scheme being evaluated. Results are shown in Fig. 4 Significant improvements of both the dispersion and dissipation properties are obtained by the TCNSs compared with WCNS-JS in intermediate and highwavenumber regimes.

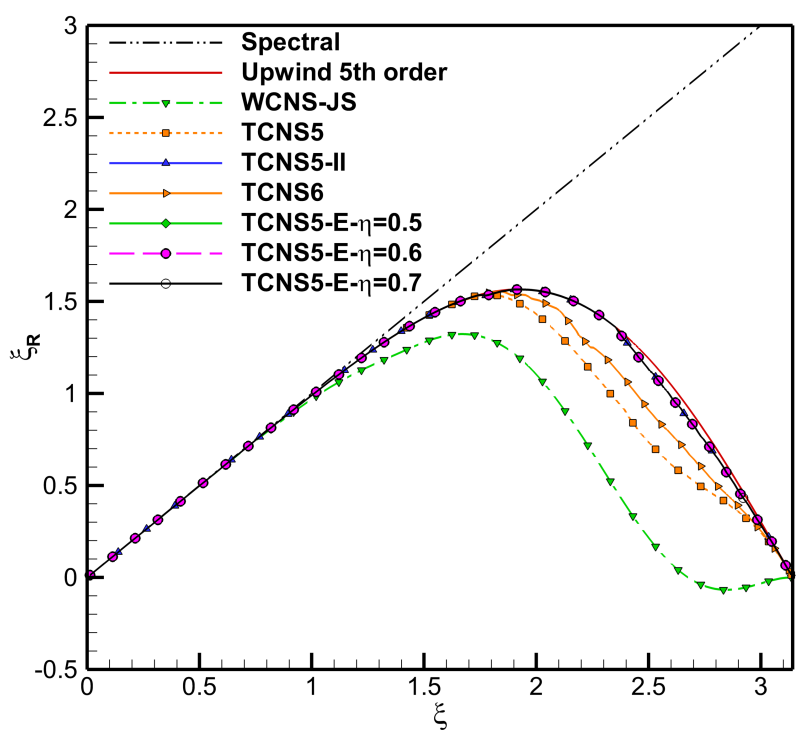

(a)

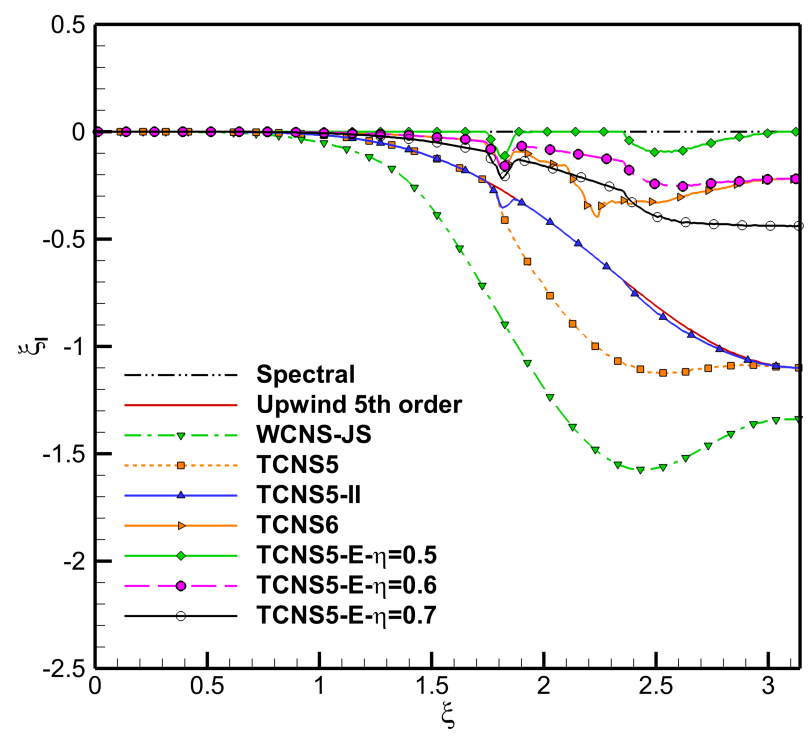

(b)

Figure 4: Approximate dispersion and dissipation properties of fifth-order schemes; (a) dispersion, and (b) dissipation.

In particular, the TCNS5-II and TCNS5-E almost fully recover the dispersion property of the underlying linear scheme, mostly due to using the adaptive strategy introduced in Section 3.2 In addition, since dispersion properties of the five-point scheme TCNS5-II and the six-point scheme TCNS5-E are essentially the same, the present TCNS5-E does not show any significant advantage in this property compared with the five-point scheme TCNS5-II. Whereas, the present TCNS5-E shows satisfying numerical dissipation property, even compared with the TCNS6. The dispersion error of the TCNS6 is a little larger compared with the TCNS5-E. The difference is produced probably due to using different parameters in the adaptive nonlinear dissipation function, which is first introduced in Ref. 31] and shown in the Appendix.

The present six-point scheme TCNS5-E showing the low-dissipation property, uses a centered stencil in the stencil-selection procedure, reducing the overall numerical dissipation. The effect of using different weights $\eta$ in Eq. (18) is also investigated. As discussed in the previous subsection 3.5, the fifth-order upwind scheme is recovered when $\eta=1.0$, and the six-order centered scheme is obtained if $\eta=0.5$. Gradually decreasing the value of weight $\eta$ from 1 to 0.5 reduces dissipation of the six-point interpolation by including the anti-dissipation effect from the downwind polynomial. A weight $\eta=0.6$ is used in all of the following numerical cases as a compromise between controlling spurious oscillations and reducing dissipation errors. In the following numerical test cases, this adaptive strategy is applied by default for both TCNS5-II and 
TCNS5-E.

\section{Numerical results}

sufficiently small to achieve temporally stable and converged results.

\subsection{Linear advection problem}

The one-dimensional Gaussian pulse advection problem 38 is used to assess the order of accuracy of the proposed schemes when solutions are smooth. This problem is modeled by the linear advection equation

$$
\frac{\partial u}{\partial t}+\frac{\partial u}{\partial x}=0, \quad x \in[0,1],
$$

with periodic boundary conditions and initial condition

$$
u(x, 0)=e^{-300\left(x-x_{c}\right)^{2}}, \quad x_{c}=0.5 .
$$

Temporal integration is performed up to $t=1$, corresponding to one period of the single wave propagation in time. Uniformly-spaced grids are progressively refined by a factor of two from the coarsest grid of $N=51$ points. As noted above, the numerical simulation on each grid is conducted using sufficiently small time steps so as to achieve temporally converged results.

Table 2 and Fig. 5 illustrate numerical errors and convergence rates of the various numerical schemes used. Accuracy of TCNS5 and TCNS5-II coincides with that of the fifth-order underlying linear scheme, indicating that the optimal linear scheme is recovered in this case. WCNS-JS also shows approximate fifthorder accuracy, but its resolution is significantly lower than that of the other three nonlinear schemes. The accuracy of TCNS5-E is of fifth-order approximately, but its resolution is even higher than the fifth-order linear scheme because of the low dissipation property of the six-point interpolation. 
Table 2: $L^{\infty}$-error and convergence rate of fifth-order schemes in solving the linear advection equation at $t=1$.

\begin{tabular}{cccccccccccc}
\hline \multirow{2}{*}{$\mathrm{N}$} & \multicolumn{2}{c}{ Linear } & \multicolumn{2}{c}{ WCNS-JS } & \multicolumn{2}{c}{ TCNS5 } & \multicolumn{2}{c}{ TCNS5-II } & \multicolumn{2}{c}{ TCNS5-E } \\
\cline { 2 - 11 } & Error & Order & Error & Order & Error & Order & Error & Order & Error & Order \\
\hline 51 & $6.74 \mathrm{E}-02$ & $*$ & 0.146572 & $*$ & $6.56 \mathrm{E}-02$ & $*$ & $6.69 \mathrm{E}-02$ & $*$ & $4.40 \mathrm{E}-02$ & $*$ \\
101 & $5.01 \mathrm{E}-03$ & 3.75 & $1.56 \mathrm{E}-02$ & 3.23 & $5.01 \mathrm{E}-03$ & 3.71 & $5.01 \mathrm{E}-03$ & 3.74 & $1.72 \mathrm{E}-03$ & 4.68 \\
201 & $1.84 \mathrm{E}-04$ & 4.77 & $6.94 \mathrm{E}-04$ & 4.49 & $1.84 \mathrm{E}-04$ & 4.77 & $1.84 \mathrm{E}-04$ & 4.77 & $4.45 \mathrm{E}-05$ & 5.27 \\
401 & $5.89 \mathrm{E}-06$ & 4.96 & $2.82 \mathrm{E}-05$ & 4.62 & $5.89 \mathrm{E}-06$ & 4.96 & $5.89 \mathrm{E}-06$ & 4.96 & $1.24 \mathrm{E}-06$ & 5.16 \\
801 & $1.85 \mathrm{E}-07$ & 4.99 & $9.91 \mathrm{E}-07$ & 4.83 & $1.85 \mathrm{E}-07$ & 4.99 & $1.85 \mathrm{E}-07$ & 4.99 & $3.76 \mathrm{E}-08$ & 5.05 \\
1601 & $5.80 \mathrm{E}-09$ & 5.00 & $3.21 \mathrm{E}-08$ & 4.95 & $5.80 \mathrm{E}-09$ & 5.00 & $5.80 \mathrm{E}-09$ & 5.00 & $1.17 \mathrm{E}-09$ & 5.01 \\
\hline
\end{tabular}

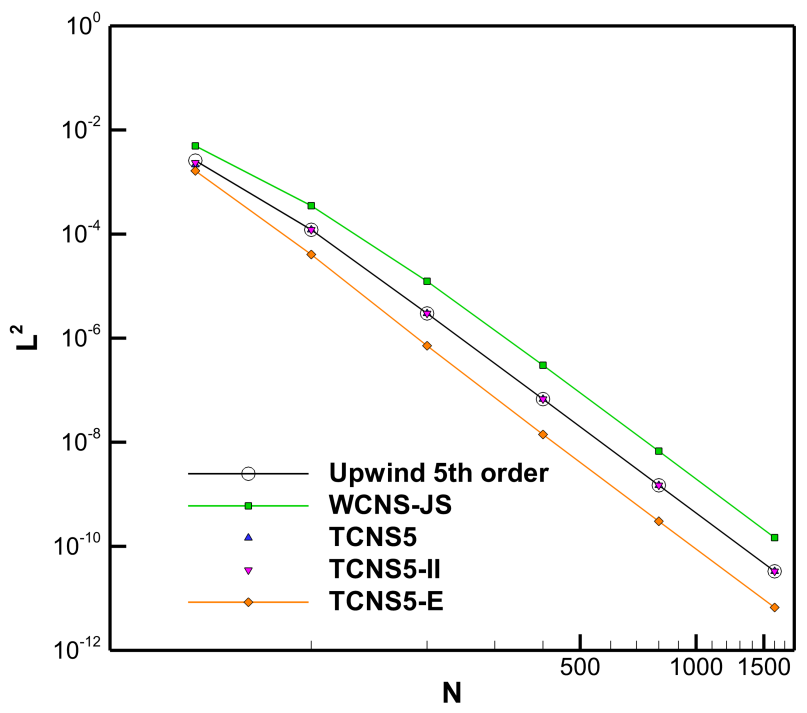

(a) $L^{2}$ error

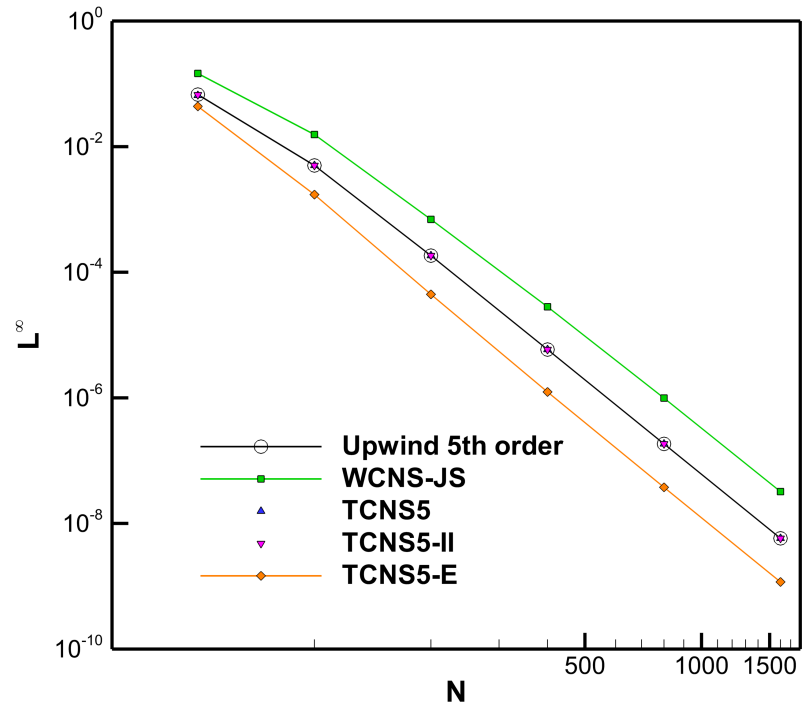

(b) $L^{\infty}$ error

Figure 5: Spatial convergence rate of fifth-order schemes at $t=1$ for $L^{2}$ - and $L^{\infty}$-errors in solutions to the linear advection equation.

\subsection{Inviscid Burgers' equation}

The one-dimensional inviscid Burgers' equation [39] is used to assess actual order of accuracy of the proposed schemes when applied to a nonlinear scalar equation. The governing equation is

$$
\frac{\partial u}{\partial t}+\frac{1}{2} \frac{\partial u^{2}}{\partial x}=0, \quad x \in[0,2] \text {, }
$$

with periodic boundary conditions and initial condition

$$
u(x, 0)=\frac{1}{2}+\sin (\pi x) .
$$

The exact solution is computed by solving the derived general characteristic relation given in [39. The solution is smooth for $0 \leq t<1 / \pi$, and a discontinuity develops and starts to interact with the expansion 


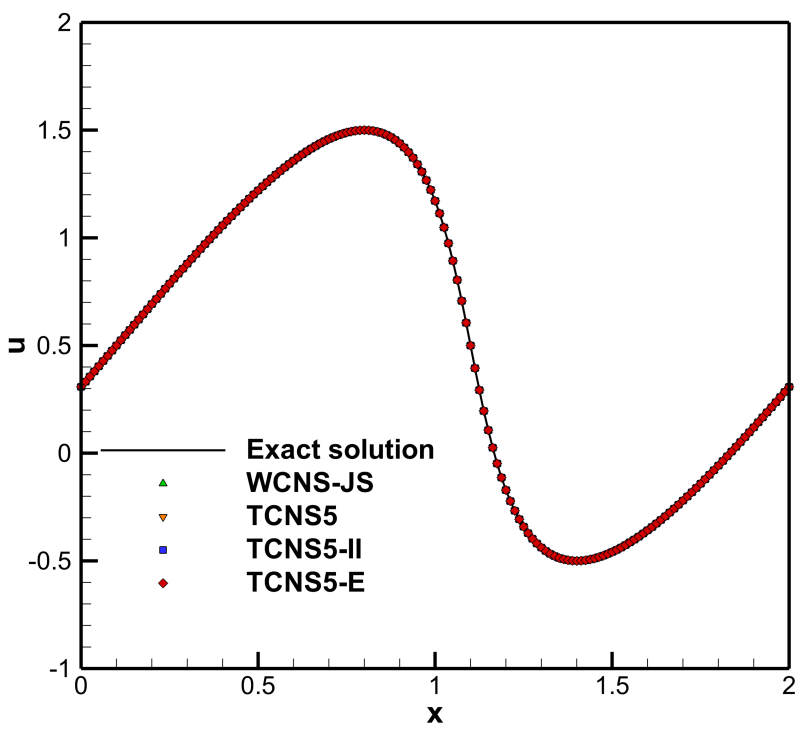

(a) $t=0.2$

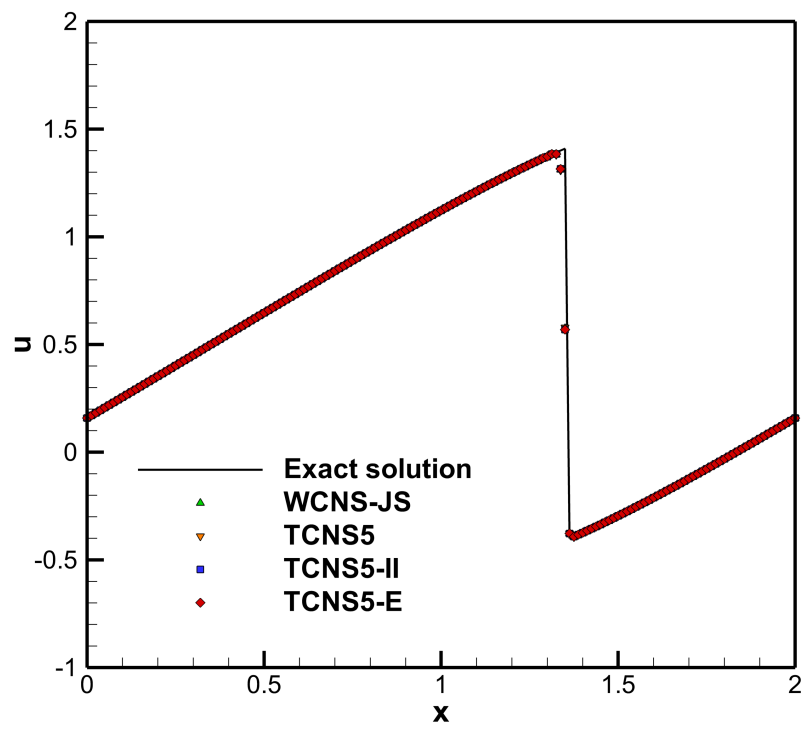

(b) $t=0.7$

Figure 6: One-dimensional inviscid Burgers' equation solution on a grid of 161 points; numerical and exact solution at (a) $t=0.2$, and $(\mathrm{b}) t=0.7$.

wave when $t=1 / \pi$. Results at $t=0.2$ and $t=0.7$ are presented to show the continuous and discontinuous parts, respectively, of the solution. Solutions computed with the above fifth-order schemes are compared with the exact solution in Fig. 6, and show good agreement for both smooth and discontinuous parts. As shown in Fig 6(b), all solutions obtained from these nonlinear schemes are free of overshoot at the discontinuity, and sharp resolution is achieved.

Moreover, $L^{\infty}$-error and convergence rate of each scheme at $t=0.2$ are presented in Table 3. Spatial accuracy of fifth order is achieved by all methods for the refined meshes except that the TCNS5-E yields results with apparent order of accuracy slightly higher than five. Perfect agreement can be found for TCNS5, TCNS5-II and the underlying fifth-order linear scheme, indicating the optimal linear schemes are recovered by both TCNS and TCNS-II. TCNS-E is more accurate than the other fifth-order schemes. This is attributed to use of the six-point stencil for polynomial construction, which is the only difference between the TCNS5-II and TCNS5-E, and thus it is expected that asymptotically (as $h \rightarrow 0$ ) fifth-order accuracy can be observed.

\subsection{Sod and Lax shock tube problems}

Riemann initial-value problems of Sod [40] and Lax [41] are used to further evaluate shock-capturing capability of the proposed schemes employing discretization of the 1-D Euler equations

$$
\begin{gathered}
\frac{\partial \rho}{\partial t}+\frac{\partial(\rho u)}{\partial x}=0 \\
\frac{\partial(\rho u)}{\partial t}+\frac{\partial\left(\rho u^{2}\right)}{\partial x}=-\frac{\partial p}{\partial x}, \\
\frac{\partial E}{\partial t}+\frac{\partial(u E)}{\partial x}=-\frac{\partial(u p)}{\partial x},
\end{gathered}
$$


Table 3: $\quad L^{\infty}$-error and convergence rate of fifth-order schemes in solving the $1-\mathrm{D}$ inviscid Burgers equation at $\mathrm{t}=0.2$.

\begin{tabular}{ccccccccccc}
\hline & \multicolumn{2}{c}{ Linear } & \multicolumn{2}{c}{ WCNS-JS } & \multicolumn{2}{c}{ TCNS5 } & \multicolumn{2}{c}{ TCNS5-II } & \multicolumn{2}{c}{ TCNS5-E } \\
\cline { 2 - 10 } & Error & Order & Error & Order & Error & Order & Error & Order & Error & Order \\
\hline 21 & $4.85 \mathrm{E}-03$ & $*$ & $3.59 \mathrm{E}-03$ & $*$ & $4.85 \mathrm{E}-03$ & $*$ & $4.85 \mathrm{E}-03$ & $*$ & $8.86 \mathrm{E}-03$ & $*$ \\
41 & $1.17 \mathrm{E}-03$ & 2.05 & $1.75 \mathrm{E}-03$ & 1.04 & $1.17 \mathrm{E}-03$ & 2.05 & $1.17 \mathrm{E}-03$ & 2.05 & $8.82 \mathrm{E}-04$ & 3.33 \\
81 & $1.15 \mathrm{E}-04$ & 3.35 & $1.78 \mathrm{E}-04$ & 3.30 & $1.15 \mathrm{E}-04$ & 3.35 & $1.15 \mathrm{E}-04$ & 3.35 & $4.24 \mathrm{E}-05$ & 4.38 \\
161 & $4.69 \mathrm{E}-06$ & 4.62 & $7.54 \mathrm{E}-06$ & 4.56 & $4.69 \mathrm{E}-06$ & 4.62 & $4.69 \mathrm{E}-06$ & 4.62 & $1.57 \mathrm{E}-06$ & 4.76 \\
321 & $1.56 \mathrm{E}-07$ & 4.91 & $2.55 \mathrm{E}-07$ & 4.89 & $1.56 \mathrm{E}-07$ & 4.91 & $1.56 \mathrm{E}-07$ & 4.91 & $3.51 \mathrm{E}-08$ & 5.48 \\
641 & $4.67 \mathrm{E}-09$ & 5.06 & $7.92 \mathrm{E}-09$ & 5.01 & $4.67 \mathrm{E}-09$ & 5.06 & $4.67 \mathrm{E}-09$ & 5.06 & $7.38 \mathrm{E}-10$ & 5.57 \\
\hline
\end{tabular}

where $E=e+\frac{1}{2} u^{2}$ is the total energy per unit mass, and $e$ is internal energy. The dependent variables are related through the perfect gas equation of state given by $p=(\gamma-1) \rho e$ with $\gamma=1.4$, thus closing the Euler equations.

The Sod shock tube problem involves a right-moving shock of Mach number 1.7, while for the Lax shock tube problem, the right-moving shock has Mach number 2.0. Initial conditions of the Sod problem are

$$
(\rho, u, p)=\left\{\begin{array}{cc}
(1,0,1) & x \in[0,0.5], \\
(0.125,0,0.1) & x \in(0.5,1],
\end{array}\right.
$$

and by solving the problem on an evenly-distributed grid of $N=101$ points the results at $t=0.2$ are given in Fig. 7. Initial conditions for the Lax shock-tube problem are

$$
(\rho, u, p)=\left\{\begin{array}{cc}
(0.445,0.698,3.528) & x \in[0,0.5], \\
(0.5,0,0.571) & x \in(0.5,1] .
\end{array}\right.
$$

This case is also simulated on an evenly distributed grid of $N=101$ points, and the results at $t=0.14$ are 


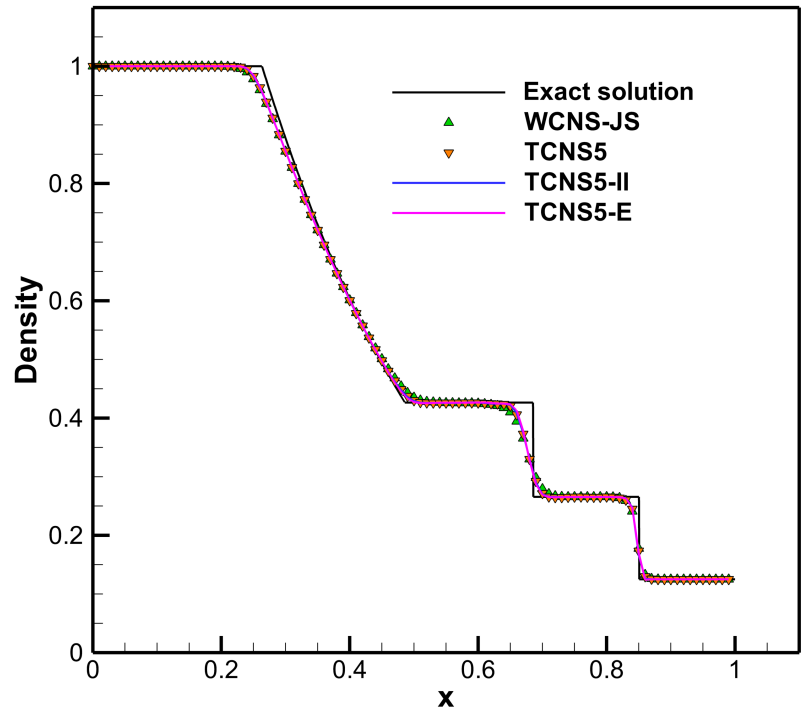

(a)

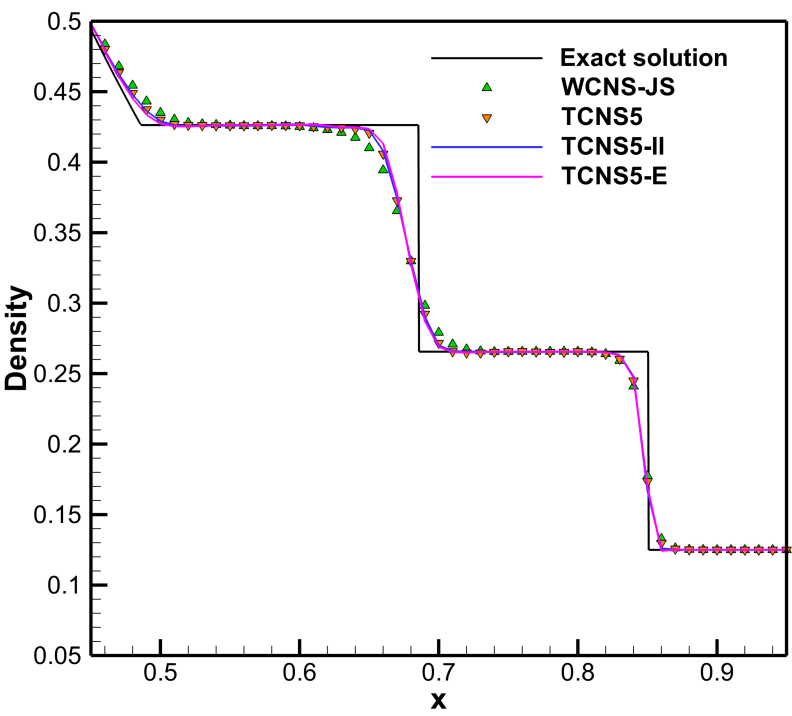

(b)

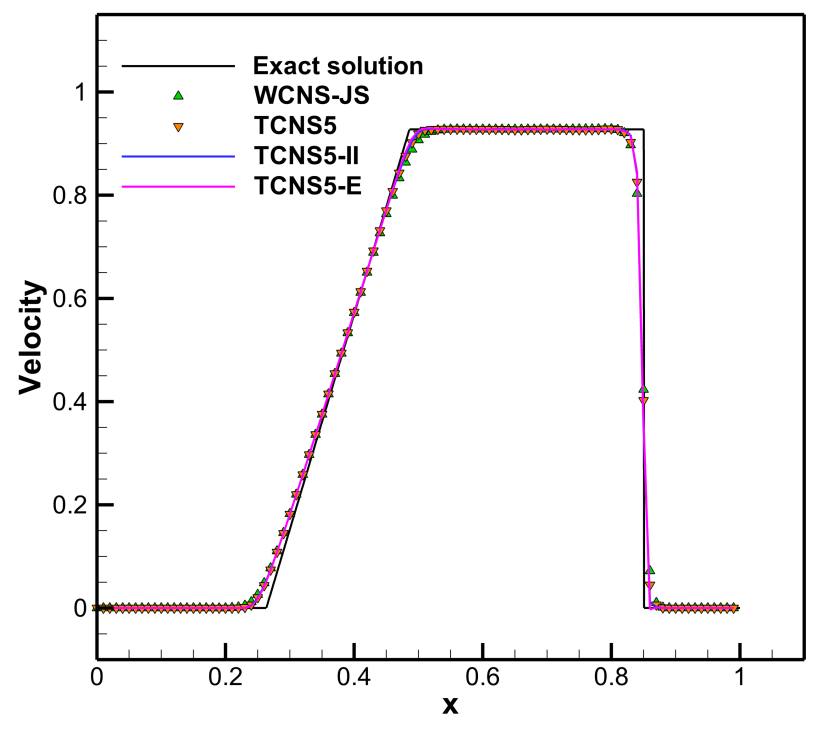

(c)

Figure 7: Numerical and exact solutions of the Sod problem at $t=0.2$; (a) density, (b) zoom in of density, (c) velocity.

scale wave structure evolves after the shock wave interacts with the oscillating density wave, and both shock-capturing and wave-resolution capabilities are evaluated for the methods considered herein via this problem.

The problem again employs the 1-D Euler equations and is initialized by

$$
(\rho, u, p)=\left\{\begin{array}{rr}
(1.515695,0.523346,1.805), & x \in[0,0.5], \\
(1+0.1 \sin (20 \pi(x-5)), 0,1), & x \in(0.5,10] .
\end{array}\right.
$$

This case is run on a grid of $N=1001$ points which are uniformly distributed, and the results at $t=5$ are 


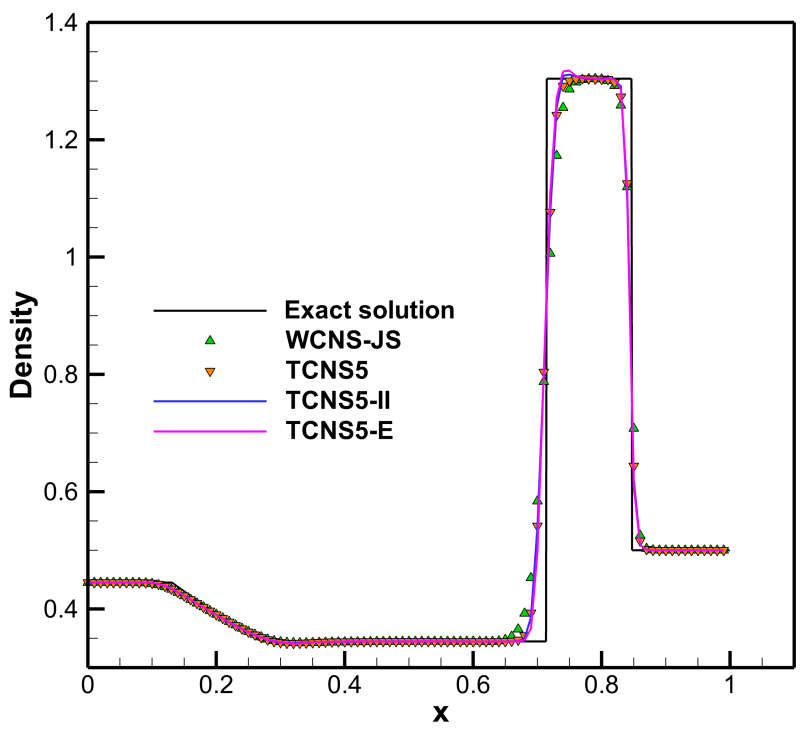

(a)

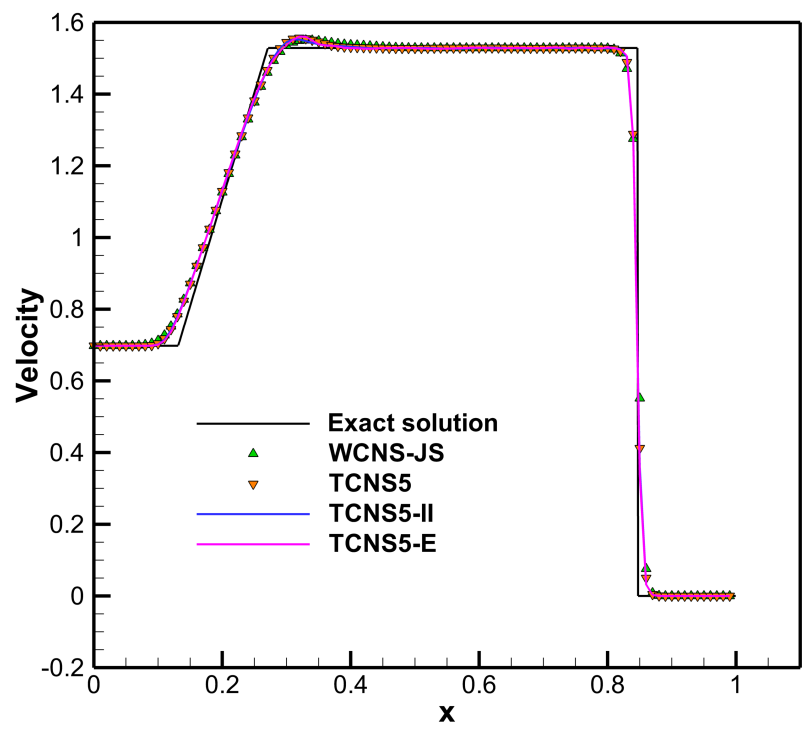

(b)

Figure 8: Numerical and exact solutions of the Lax problem at $t=0.14$; (a) density, and (b) velocity.

given. Since there is not a theoretical solution for this problem, the numerical result calculated by using the WCNS-JS on a grid of $N=5001$ points is used as a reference solution.

As shown in Fig. 9, TCNSs produce considerably better resolved density waves behind the shock wave compared with the WCNS-JS. The result of the WCNS-JS indicates strong numerical dissipation, since small-scale wave structures are relatively smeared. More specifically, two six-point schemes, viz. TCNS5-E and TCNS6, show better resolution compared with two five-point TCNSs. Therefore, one may conclude that using the six-point low-dissipation stencil indeed dominates the performance of the present TCNS5-E, although the smoothness measurement is simple. Furthermore, in Table 4 we can see that the TCNS5-E is as efficient as the TCNS5-II, and less time consuming compared with the TCNS6. 


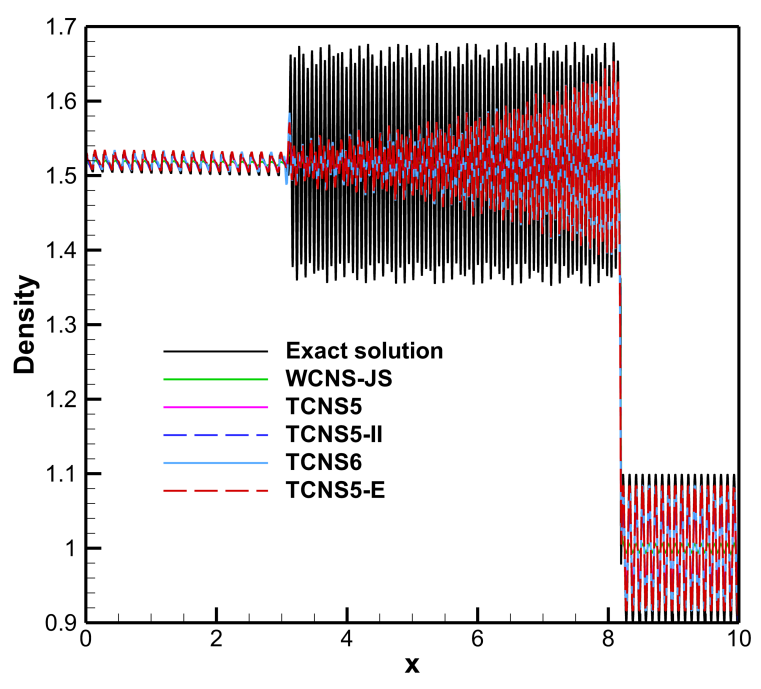

(a)

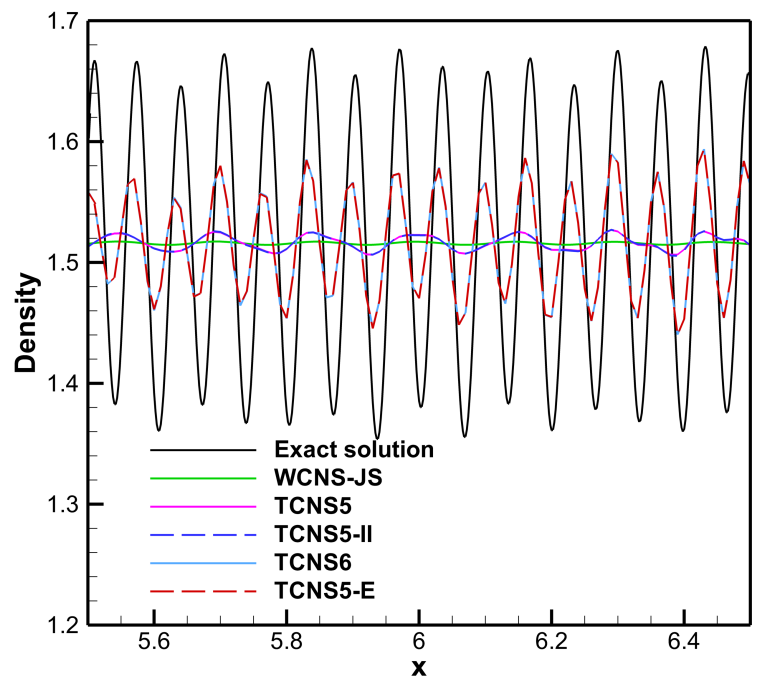

(b)

Figure 9: Shock/density-wave interaction problem; numerical solutions and the exact solution at $t=5$; (a) full spatial domain, and (b) zoom in on high-amplitude region. Riemann solver in place of the dissipative Rusanov scheme. However, developing or evaluating different upwind flux schemes is beyond the scope of the present research, and will not be discussed further in the following sections.

Table 4: CPU timing (in seconds) per time step in the shock/density-wave interaction problem.

\begin{tabular}{cccccc}
\hline Grid size & WCNS-JS & TCNS5 & TCNS5-II & TCNS6 & TCNS5-E \\
\hline 1001 & 0.02 & 0.03 & 0.04 & 0.08 & 0.04 \\
\hline
\end{tabular}

\subsection{Two-dimensional Riemann problems}

In this section, the configurations 3 and 6 out of 19 2-D Riemann problems used by Lax and Liu [43] are employed to evaluate performance of the currently investigated numerical schemes. In particular, these two configurations are characterized by rich small scales in the flow fields, and thus are specifically chosen to evaluate ability of the proposed numerical schemes to resolve fine flow structures. These 2-D Riemann problems are widely-solved canonical problems in the present context, analogous to the 1-D Sod shock 
straightforward extension of the 1-D case given above:

$$
\begin{aligned}
\frac{\partial \rho}{\partial t}+\frac{\partial(\rho u)}{\partial x}+\frac{\partial(\rho v)}{\partial y} & =0 \\
\frac{\partial(\rho u)}{\partial t}+\frac{\partial\left(\rho u^{2}\right)}{\partial x}+\frac{\partial(\rho u v)}{\partial y} & =-\frac{\partial p}{\partial x}, \\
\frac{\partial(\rho v)}{\partial t}+\frac{\partial(\rho u v)}{\partial x}+\frac{\partial\left(\rho v^{2}\right)}{\partial y} & =-\frac{\partial p}{\partial y}, \\
\frac{\partial E}{\partial t}+\frac{\partial(u E)}{\partial x}+\frac{\partial(v E)}{\partial y} & =-\frac{\partial(u p)}{\partial x}-\frac{\partial(v p)}{\partial y} .
\end{aligned}
$$

\subsubsection{Configuration 3}

Initial conditions for configuration 3 are given by

$$
(\rho, u, v, p)=\left\{\begin{array}{cl}
(1.5,0,0,1.5) & (x, y) \in\left[\frac{1}{2}, 1\right] \times\left[\frac{1}{2}, 1\right], \\
(0.5323,1.206,0,0.3) & (x, y) \in\left[0, \frac{1}{2}\right] \times\left[\frac{1}{2}, 1\right], \\
(0.138,1.206,1.206,0.029) & (x, y) \in\left[0, \frac{1}{2}\right] \times\left[0, \frac{1}{2}\right], \\
(0.5323,0,1.206,0.3) & (x, y) \in\left[\frac{1}{2}, 1\right] \times\left[0, \frac{1}{2}\right] .
\end{array}\right.
$$

Boundary conditions are

$$
\begin{aligned}
& \frac{\partial \mathbf{u}(x, y, t)}{\partial x}=0, \quad x=0,1, \quad \forall y, t, \\
& \frac{\partial \mathbf{u}(x, y, t)}{\partial y}=0, \quad y=0,1, \quad \forall x, t,
\end{aligned}
$$

where the vector of primitive variables is given by $\mathbf{u}=(\rho, u, v, p)^{T}$. It should be noted that computations are terminated before the shock structures interacted with boundaries, and thus the discussion will focus on the central part of the flow field.

Figure 10 presents results obtained with $1024^{2}$ grid points with uniform spacing using WCNS-JS and TCNSs, at $t=0.3$. All TCNSs capture more vortices along contact lines than does WCNS-JS. The solution computed with TCNS5-II is slightly different from that of TCNS5, and TCNS5-E resolves a few unique small features not seen in the other figures, probably due to its low numerical dissipation. Especially, the present TCNS5-E shows similar resolution compared with the TCNS6 which uses the traditional "convex combination" concept for the nonlinear interpolation.

Moreover, a significant advantage of the present TCNS5-E is shown in Table 5 . It can be found that the CPU time of using the TCNS5-E is only slightly higher than using the TCNS5-II, which is expectable since the algorithms of these two schemes are almost the same. However, the present TCNS5-E is more

efficient compared with the TCNS6, since the smoothness measurement is simpler while using the extended strategy introduced in section 3.4. Therefore, we may conclude that the present TCNS5-E achieves similar performance but requires less computational resource, compared with the TCNS6.

Without physical results for comparisons, one must question whether this numerically-produced "turbulence" actually represents physics. In fact, Shi et al. 44] suggest that during a mesh refinement slip lines 
Table 5: CPU timing (in seconds) per time step in the 2D Riemann problem of configuration 3

\begin{tabular}{cccccc}
\hline Grid size & WCNS-JS & TCNS5 & TCNS5-II & TCNS6 & TCNS5-E \\
\hline $1024 \times 1024$ & 3.72 & 4.95 & 7.35 & 15.06 & 7.54 \\
\hline
\end{tabular}

\subsection{Rayleigh-Taylor instability}

The Rayleigh-Taylor instability problem, which contains both discontinuities and complex flow structures, has been widely studied, both experimentally and computationally, and it is used here as a final example

Boundary conditions are the same as in the previous test case.

Results at $t=0.3$ are shown in Fig. 11. All TCNSs resolve abundant small-scale flow structures along contact lines. Minor differences can be seen between the TCNS5-II result and that of TCNS5, and two six-point schemes yield solutions characterized with much richer small-scale structures. Apparently, the TCNS5-II which uses the adaptive nonlinear dissipation, is low-dissipative compared with the TCNS5, and the six-point stencil used in the TCNS5-E further reduces the overall numerical dissipation, thus resulting this low-dissipation scheme. The results of the TCNS5-E and TCNS6 are similar. However, as shown in Table 6, the TCNS5-E with using the present extended stencil is more efficient than the TCNS6. Of course, in the absence physical results or demonstrably accurate simulations, it is somewhat difficult to rank these procedures in modelling real physics. 
$\rho: \quad 0.10 .20 .30 .50 .60 .70 .80 .91 .01 .21 .31 .41 .51 .61 .7$
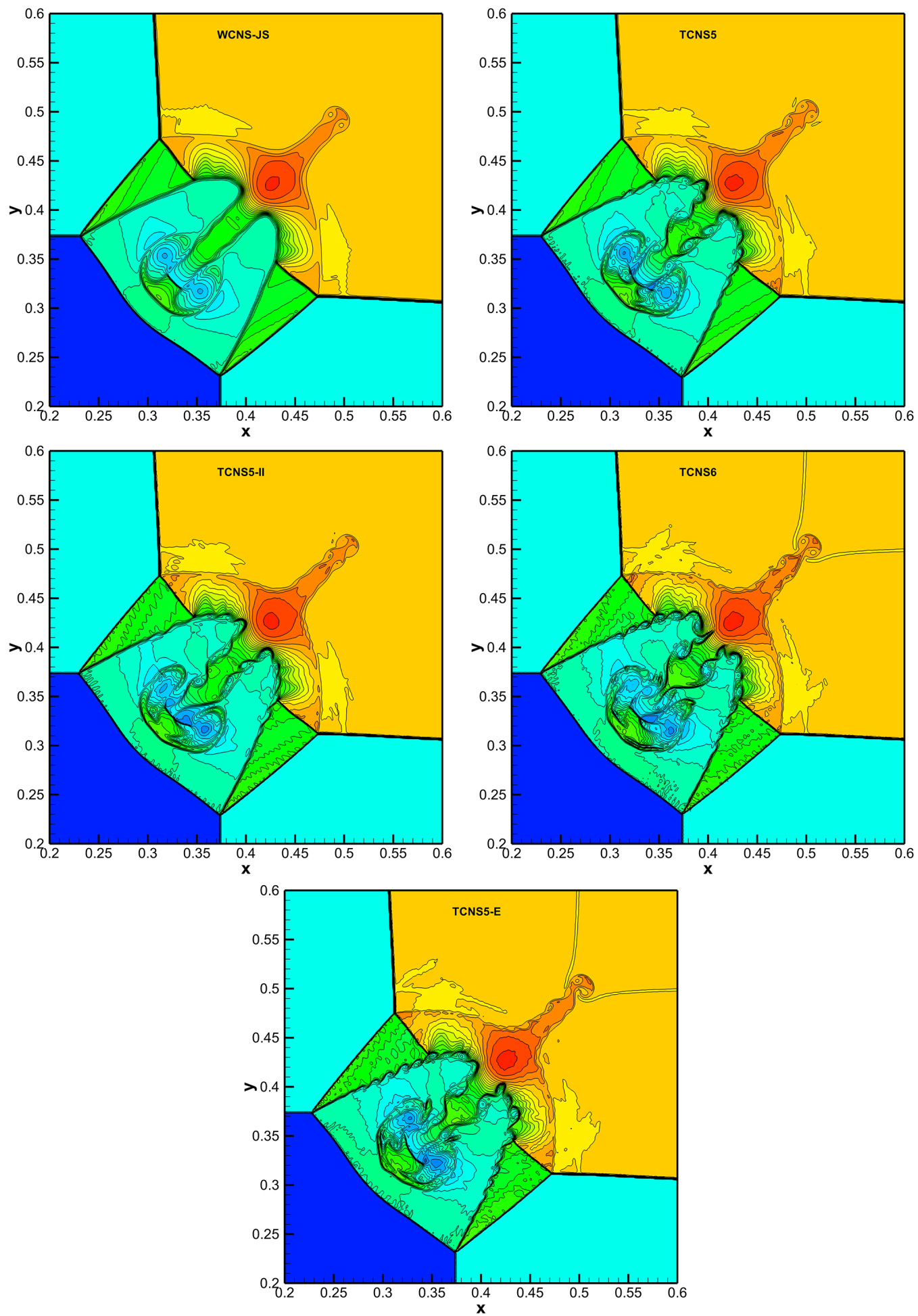

Figure 10: Configuration 3 of 2-D Riemann problems in [43; 30 density contour lines ranging from 0.1 to 1.8 at $t=0.3$. 

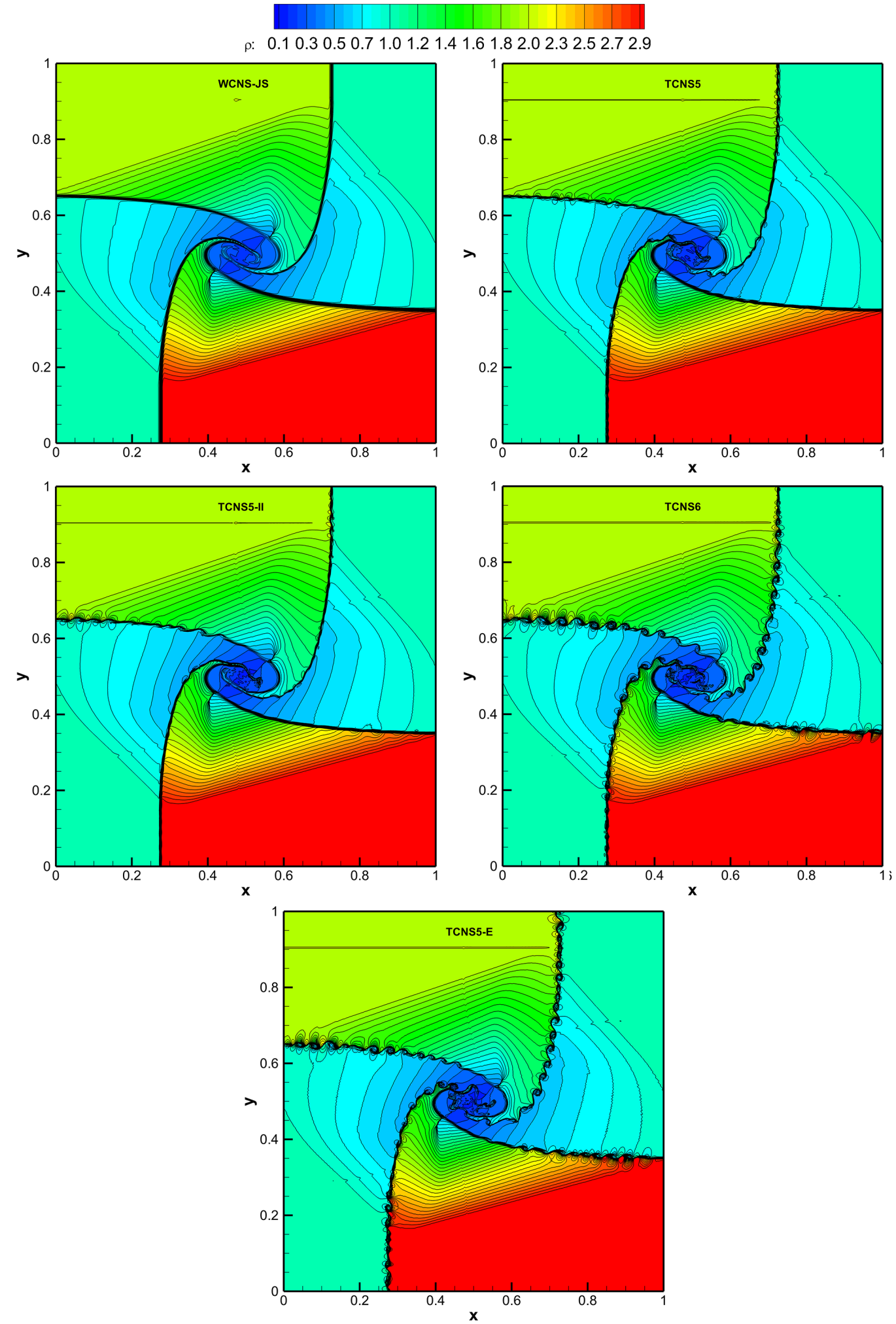

Figure 11: Configuration 6 of 2-D Riemann problems in 43] computed on a grid of $1024 \times 1024$ points; 40 density contour lines ranging from 0.1 to 2.9 at $t=0.3$. 
Table 6: CPU timing (in seconds) per time step in the 2D Riemann problem of configuration 6

\begin{tabular}{cccccc}
\hline Grid size & WCNS-JS & TCNS5 & TCNS5-II & TCNS6 & TCNS5-E \\
\hline $1024 \times 1024$ & 3.75 & 4.96 & 7.43 & 15.03 & 7.58 \\
\hline
\end{tabular}

for examining performance of the presently-studied methods. Again, the 2-D Euler equations comprise the basic mathematical model. In the present case, initial conditions are given by

$$
(\rho, u, v, p)= \begin{cases}(2,0,-0.025 a \cos (8 \pi x), 1+2 y) & (x, y) \in[0,0.25] \times[0,0.5) \\ (1,0,-0.025 a \cos (8 \pi x), y+3 / 2) & (x, y) \in[0,0.25] \times[0.5,1]\end{cases}
$$

where $a$ is the speed of sound, given by $a=\sqrt{\gamma p / \rho}$ and a different $\gamma=\frac{5}{3}$ is used for this specific case. Reflecting boundary conditions are imposed at the left- and right-hand sides of the domain. Constant boundary conditions are given for the top and bottom sides, expressed as

$$
(\rho, u, v, p)=\left\{\begin{array}{cc}
(1,0,0,2.5) & y=1, \quad \forall t, x \\
(2,0,0,1) & y=0, \quad \forall t, x
\end{array}\right.
$$

Two source terms, $\rho$ and $\rho v$, are added to the right-hand side of the third and the fourth equations, respectively, of the 2-D Euler system given at the beginning of this subsection, as done by Shi et al. [44, among others. (Note: this does not correctly adhere to dimensional consistency of terms in the equations of motion, so any correspondence with actual physics would be accidental. Moreover, the Rayleigh-Taylor problem is 3D, so again, not much actual physics can be expected from these simulations, but only numerical properties are investigated.) Two grids were used, namely, $128 \times 512$ and $256 \times 1024$ points. Density profiles at the final time $t=1.95$, computed using TCNS5, TCNS5-II and TCNS5-E, are shown in Fig. 12 , It is rather clear that grid-function convergence, in any usual sense, is not occurring for any of the methods. 


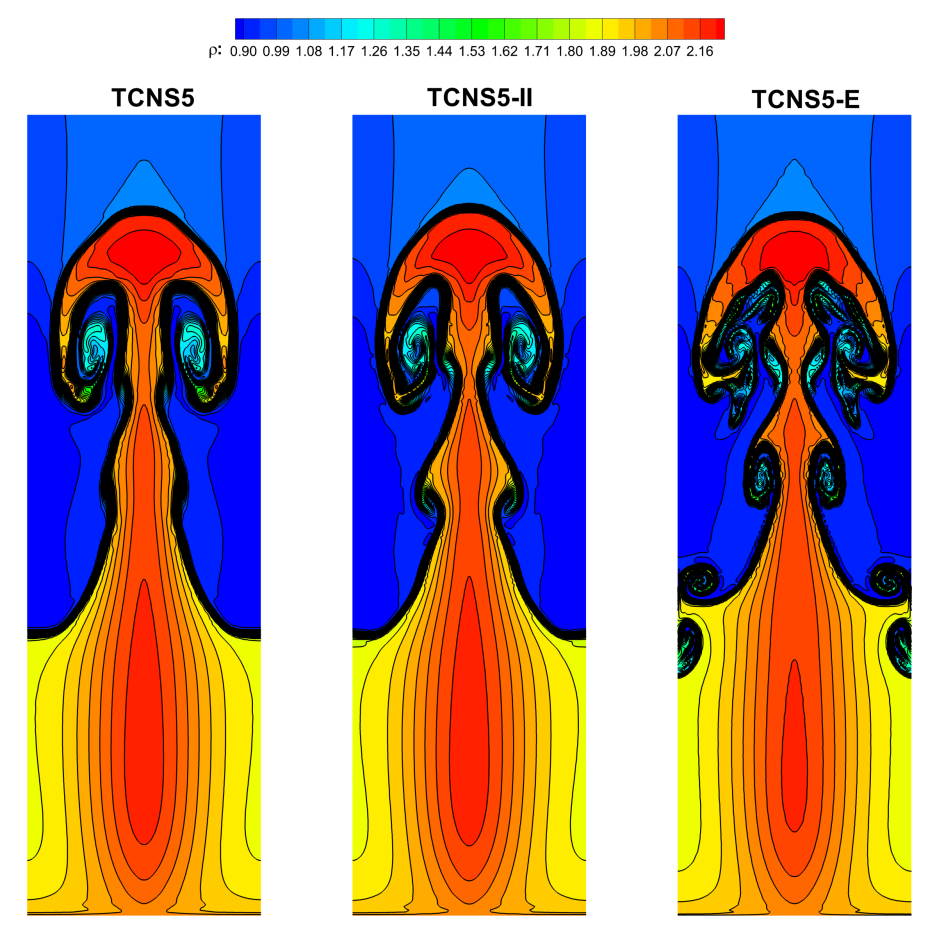

(a) Grid resolution $128 \times 512$

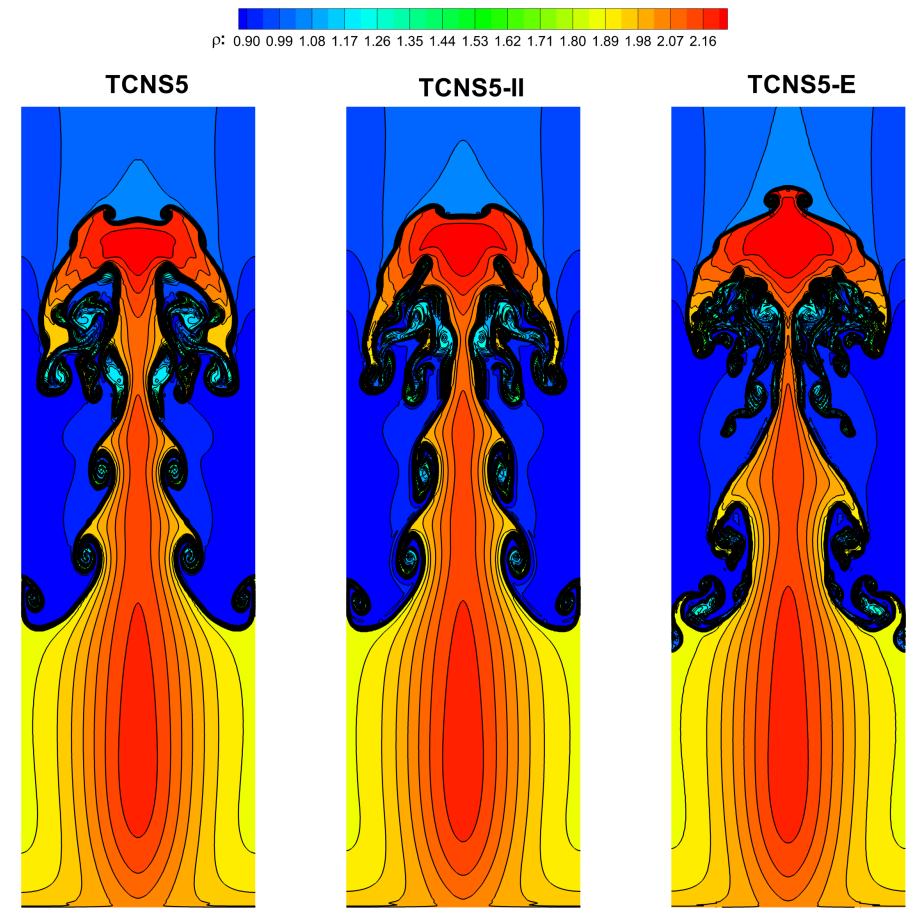

(b) Grid resolution $256 \times 1024$

Figure 12: Rayleigh-Taylor instability problem; 30 density contour lines ranging from 0.9 to 2.2 . 
II, demonstrating the advantage of the low numerical dissipation of the TCNS5-E. The performance of TCNS5-II lies between that of TCNS5 and TCNS5-E. Moreover, symmetric density profiles are produced with all three different schemes, as should be expected. It is worth noting again, that for this problem, a more accurate Riemann solver would be quite helpful for improving resolution of the present simulations, as investigated by San and Kara [46] for a somewhat different problem.

\section{Conclusions}

Following the fundamental concept of compact nonlinear schemes, a new extending strategy is introduced to implement a six-point centered stencil by making full use of currently available five-point smoothness measurements, and numerical performance can be significantly improved without applying more complex smoothness indicators. Essentially, the smoothness measurement for the extending strategy simply acts as a discontinuity-location detector. With the smoothness/discontinuity-location information provided, an extended high-order, carefully optimized interpolation can be directly applied by using the improved stencilselection procedure described in this work. It is shown that using this specific stencil-selection procedure, instead of the traditional concept of "convex combination" yields more flexibility in development of nonlinear interpolations required to construct higher-order methods.

In order to further improve the spectral property of the proposed schemes, a mature adaptive dissipation control can be applied to control the magnitude of the cut-off parameter $C_{T}$, although the value of this parameter was first taken to be constant. Various numerical analyses have shown that the presented schemes are capable of simulating complex flow problems, including shock waves and hydrodynamic instabilities, for instance, the Rayleigh-Taylor instability problem. Results are significantly improved compared with the traditional WCNS approach. Most importantly, the present TCNS5-E is significantly more efficient than the TCNS6 which uses the traditional "convex combination" concept for the nonlinear interpolation.

\section{Acknowledgments}

This work is partly supported by the National Numerical Wind Tunnel Project (Grant No. NNW2018ZT4B09) and the National Natural Science Foundation of China (Grant No. 11872144).

Appendix A. The six-point nonlinear interpolation procedure for compact nonlinear schemes

Following the idea of Ref. 21, a six-point nonlinear interpolation procedure for TCNS can be designed based on the six-point scheme in Ref. [31, and the comparison can be made specifically for the new features introduced in the TCNS5-E. In the scheme introduced in this appendix, which is denoted as TCNS6, the

traditional "convex combination" concept is used for its nonlinear interpolation, and the numerical flux scheme or the midpoint-to-node centered flux differencing mentioned in previous sections is directly applied. 
In order to measure the smoothness of sub-stencils and calculate the nonlinear weights of the convex combination procedure, the smoothness measurement needs to be given at first. The local smoothness indicators are given in a unified form

$$
\beta_{k}=\left(h u_{i, k}^{(1)}\right)^{2}+\left(h^{2} u_{i, k}^{(2)}\right)^{2}+\left(h^{3} u_{i, k}^{(3)}\right)^{2} .
$$

Therefore, besides the Eqs. 9] and 10, we need to provide extra information, including

$$
\begin{aligned}
u_{i, 3}^{(1)} & =\frac{1}{6 h}\left(-11 u_{i}+18 u_{i+1}-9 u_{i+2}+2 u_{i+3}\right), \\
u_{i, 3}^{(2)} & =\frac{1}{h^{2}}\left(2 u_{i}-5 u_{i+1}+4 u_{i+2}-u_{i+3}\right), \\
u_{i, 3}^{(3)} & =\frac{1}{h^{3}}\left(-u_{i}+3 u_{i+1}-3 u_{i+2}+u_{i+3}\right) .
\end{aligned}
$$

It should be noted that $u_{i, 0}^{(3)}, u_{i, 1}^{(3)}$ and $u_{i, 2}^{(3)}$ are all zero.

Then the high-order global smoothness indicator can be defined based on the local smoothness indicators, resulting

$$
\tau_{6}=\left|\beta_{6}-\frac{1}{6}\left(\beta_{0}+4 \beta_{1}+\beta_{2}\right)\right|
$$

where

$$
\beta_{6}=\left(h u_{i, 6}^{(1)}\right)^{2}+\left(h^{2} u_{i, 6}^{(2)}\right)^{2}+\left(h^{3} u_{i, 6}^{(3)}\right)^{2}+\left(h^{4} u_{i, 6}^{(4)}\right)^{2}+\left(h^{5} u_{i, 6}^{(5)}\right)^{2} .
$$

Corresponding definitions used for this high-order smoothness indicator are given as

$$
\begin{aligned}
u_{i, 6}^{(1)} & =\frac{1}{60 h}\left(3 u_{i-2}-30 u_{i-1}-20 u_{i}+60 u_{i+1}-15 u_{i+2}+2 u_{i+3}\right), \\
u_{i, 6}^{(2)} & =\frac{1}{12 h^{2}}\left(-u_{i-2}+16 u_{i-1}-30 u_{i}+16 u_{i+1}-u_{i+2}\right), \\
u_{i, 6}^{(3)} & =\frac{1}{4 h^{3}}\left(-u_{i-2}-u_{i-1}+10 u_{i}-14 u_{i+1}+7 u_{i+2}-u_{i+3}\right), \\
u_{i, 6}^{(4)} & =\frac{1}{h^{4}}\left(u_{i-2}-4 u_{i-1}+6 u_{i}-4 u_{i+1}+u_{i+2}\right), \\
u_{i, 6}^{(5)} & =\frac{1}{h^{5}}\left(-u_{i-2}+5 u_{i-1}-10 u_{i}+10 u_{i+1}-5 u_{i+2}+u_{i+3}\right) .
\end{aligned}
$$

With using the present smoothness indicators, the formulas shown in section 3 including Eqs. 12, 13. and 14, can be calculated. It should be noted that the original $\tau_{5}$ shall be replaced by $\tau_{6}$. The convex combination can thus be given as

$$
u_{L, i+\frac{1}{2}}=\sum_{k=0}^{3} \omega_{k} u_{L, i+\frac{1}{2}, k}
$$

and

$$
\omega_{k}=\frac{d_{k} \delta_{k}}{\sum_{k=0}^{3} d_{k} \delta_{k}},
$$

where

$$
\begin{aligned}
& u_{L, i+\frac{1}{2}, 0}=\mathrm{P}_{5}, \\
& u_{L, i+\frac{1}{2}, 1}=\mathrm{P}_{4}, \\
& u_{L, i+\frac{1}{2}, 2}=\mathrm{P}_{3}, \\
& u_{L, i+\frac{1}{2}, 3}=\frac{1}{16}\left(5 u_{i}+15 u_{i+1}-5 u_{i+2}+u_{i+3}\right) .
\end{aligned}
$$


and

$$
d_{0}=\frac{1}{32}, d_{1}=\frac{15}{32}, \quad d_{2}=\frac{5}{16}, \quad d_{3}=\frac{3}{16} .
$$

In Eq. 18, the six-point interpolation is optimized to balance the numerical dissipation and dispersion. Here, this optimized interpolation is directly incorporated into the nonlinear interpolation procedure, resulting

$$
u_{L, i+\frac{1}{2}}=\left\{\begin{array}{cc}
\mathrm{P}_{6, L}, & \text { if } \quad \delta_{k}=1, \quad k=0, \cdots, 3, \\
\sum_{k=0}^{3} \omega_{k} u_{L, i+\frac{1}{2}, k}, & \text { otherwise. }
\end{array}\right.
$$

Finally, according to Ref. 31], part of the parameters of the adaptive procedure introduced in section 3.2 need to be redefined for the present six-point scheme. Thereinto, $\eta_{i+\frac{1}{2}}^{(6)}=\min \left(\eta_{i-1}, \eta_{i}, \eta_{i+1}, \eta_{i+2}\right), C_{r}^{(6)}=$ $0.17, \alpha_{1}^{(6)}=10.5$ and $\alpha_{2}^{(6)}=4.5$. The other parameters are directly reused without further modifications and discussion.

\section{References}

[1] X. Deng, H. Zhang, Developing high-order weighted compact nonlinear schemes, Journal of Computational Physics 165 (1) (2000) 22 - 44. doi:10.1006/jcph.2000.6594

[2] S. Pirozzoli, Numerical methods for high-speed flows, Annual Review of Fluid Mechanics 43 (1) (2011) 163-194. doi:10.1146/annurev-fluid-122109-160718.

[3] P. Tsoutsanis, I. W. Kokkinakis, L. Knzsy, D. Drikakis, R. J. R. Williams, D. L. Youngs, Comparison of structured- and unstructured-grid, compressible and incompressible methods using the vortex pairing problem, Computer Methods in Applied Mechanics and Engineering 293 (2015) 207-231. doi:10.1016/ j.cma.2015.04.010.

[4] H. Dong, L. Fu, F. Zhang, Y. Liu, J. Liu, Detonation simulations with a fifth-order TENO scheme, Communications in Computational Physics 25 (2019) 1357-1393. doi:10.4208/cicp.0A-2018-0008.

[5] G. Zhao, M. Sun, A. Memmolo, S. Pirozzoli, A general framework for the evaluation of shock-capturing schemes, Journal of Computational Physics 376 (2019) 924-936. doi:10.1016/j.jcp.2018.10.013.

[6] Z. Wang, K. Fidkowski, R. Abgrall, F. Bassi, D. Caraeni, A. Cary, H. Deconinck, R. Hartmann, K. Hillewaert, H. Huynh, N. Kroll, G. May, P.-O. Persson, B. van Leer, M. Visbal, High-order cfd methods: current status and perspective, Int. J. Numer. Meth. Fluids 72 (8) (2013) 811-845.

[7] X. Deng, H. Maekawa, Compact high-order accurate nonlinear schemes, Journal of Computational Physics 130 (1) (1997) 77 - 91. doi:10.1006/jcph.1996.5553. 
[8] S. K. Lele, Compact finite difference schemes with spectral-like resolution, Journal of Computational Physics 103 (1) (1992) 16 - 42. doi:10.1016/0021-9991(92)90324-R.

[10] T. Nonomura, K. Fujii, Robust explicit formulation of weighted compact nonlinear scheme, Computers

[17] Z. Yan, H. Liu, M. Mao, H. Zhu, X. Deng, New nonlinear weights for improving accuracy and resolution compfluid.2016.01.005

[18] S. Zheng, X. Deng, D. Wang, C. Xie, A parameter-free $\epsilon$-adaptive algorithm for improving weighted compact nonlinear schemes, Int J Numer Meth Fluids 90 (5). doi:10.1002/fld.4719

[19] H. Zhang, G. Wang, F. Zhang, Development of a multi-resolution weighted compact nonlinear scheme for hyperbolic conservation laws, International Journal of Computational Fluid Dynamics.

[20] L. Fu, X. Y. Hu, N. A. Adams, A family of high-order targeted ENO schemes for compressible-fluid simulations, Journal of Computational Physics 305 (2016) 333-359. 
[21] H. Zhang, F. Zhang, C. Xu, Towards optimal high-order compact schemes for simulating compressible flows, Applied Mathematics and Computation 355 (2019) 221-237. doi:10.1016/j.amc.2019.03.001

[22] X. Deng, High-order accurate dissipative weighted compact nonlinear schemes, Science in China Series A: Mathematics 45 (3) (2002) 356. doi:10.1360/02ys9037.

[23] X. Deng, X. Liu, M. Mao, H. Zhang, Investigation on weighted compact fifth-order nonlinear scheme and applications to complex flow, in: 17th AIAA Computational Fluid Dynamics Conference, AIAA 2005-5246, Toronto, Ontario Canada, 2005. doi:10.2514/6.2005-5246

[24] T. Nonomura, K. Fujii, Effects of difference scheme type in high-order weighted compact nonlinear schemes, Journal of Computational Physics 228 (10) (2009) 3533 - 3539. doi:10.1016/j.jcp.2009. 02.018

[25] F. Zhang, J. Liu, H. Zhang, C. Xu, An extending strategy based on teno framework for hyperbolic conservation laws, arXiv:1808.00037v4 [physics.comp-ph] (2018).

[26] F. Zhang, C. Xu, J. Liu, A simple extending strategy for TENO scheme: scalar equation and Euler equations, in: HiSST: International Conference on High-Speed Vehicle Science Technology, Moscow, Russia, 2018.

[27] L. Fu, X. Y. Hu, N. A. Adams, A new class of adaptive high-order targeted eno schemes for hyperbolic conservation laws, Journal of Computational Physics 374 (2018) 724-751. doi:10.1016/j.jcp.2018. 07.043 .

[28] S. Pirozzoli, On the spectral properties of shock-capturing schemes, Journal of Computational Physics 219 (2) (2006) 489 - 497. doi:10.1016/j.jcp.2006.07.009.

[29] S. Gottlieb, C. Shu, E. Tadmor, Strong stability-preserving high-order time discretization methods, SIAM Review 43 (1) (2001) 89-112. doi:10.1137/S003614450036757X

[30] X. Deng, Y. Shimizu, F. Xiao, A fifth-order shock capturing scheme with two-stage boundary variation 口 diminishing algorithm, Journal of Computational Physics 386 (2019) 323-349. doi:10.1016/j.jcp. 2019.02 .024

[31] L. Fu, X. Y. Hu, N. A. Adam, Improved five- and six-point targeted essentially nonoscillatory schemes with adaptive dissipation, AIAA JOURNAL 57 (3) (2019) 1143-1158. doi:10.2514/1. J057370.

[32] Y.-X. Ren, M. Liu, H. Zhang, A characteristic-wise hybrid compact-weno scheme for solving hyperbolic conservation laws, Journal of Computational Physics 192 (2) (2003) 365 - 386. doi:10.1016/j.jcp. 2003.07 .006 
[33] S. Clain, S. Diot, R. Loubère, A high-order finite volume method for systems of conservation lawsmulti-dimensional optimal order detection (mood), Journal of Computational Physics 230 (10) (2011) 4028 - 4050. doi:10.1016/j.jcp.2011.02.026

[34] J. Fernndez-Fidalgo, X. Nogueira, L. Ramrez, I. Colominas, An a posteriori, efficient, high-spectral resolution hybrid finite-difference method for compressible flows, Computer Methods in Applied Mechanics and Engineering 335 (2018) 91-127. doi:10.1016/j.cma.2018.02.013

[35] V. V. Rusanov, Calculation of interaction of non-stationary shock waves with obstacles, Zh. Vychisl. Mat. Mat. Fiz. 1 (2) (1961) 267-279.

[36] F. Zhang, J. Liu, B. Chen, W. Zhong, Evaluation of rotated upwind schemes for contact discontinuity and strong shock, Computers \& Fluids 134-135 (2016) 11-22.

[37] F. Zhang, J. Liu, B. Chen, W. Zhong, A robust low-dissipation AUSM-family scheme for numerical shock stability on unstructured grids, Int. J. Numer. Meth. Fluids 84 (2017) 135-151. doi:10.1002/f1d.4341

[38] C.-W. Shu, S. Osher, Efficient implementation of essentially non-oscillatory shock-capturing schemes,ii, J. Comput. Phys. 83 (1) (1989) 32-78. doi:10.1016/0021-9991(89)90222-2.

[39] A. Harten, B. Engquist, S. Osher, S. R. Chakravarthy, Uniformly high order accurate essentially nonoscillatory schemes, III, Journal of Computational Physics 71 (2) (1987) 231-303.

[40] G. A. Sod, A survey of several finite difference methods for systems of nonlinear hyperbolic conservation laws, Journal of Computational Physics 27 (1) (1978) 1-31.

[41] P. D. Lax, Weak solutions of nonlinear hyperbolic equations and their numerical computation, Communications on Pure and Applied Mathematics 7 (1) (1954) 159-193. doi:10.1002/cpa.3160070112.

[42] V. Titarev, E. Toro, Finite-volume WENO schemes for three-dimensional conservation laws, Journal of Computational Physics 201 (2004) 238-260.

[43] P. Lax, X. Liu, Solution of two-dimensional riemann problems of gas dynamics by positive schemes, SIAM Journal on Scientific Computing 19 (2) (1998) 319-340. doi:10.1137/S1064827595291819.

[44] J. Shi, Y.-T. Zhang, C.-W. Shu, Resolution of high order weno schemes for complicated flow structures, Journal of Computational Physics 186 (2) (2003) 690 - 696. doi:10.1016/S0021-9991(03)00094-9.

[45] G. Zhao, M. Sun, S. Xie, H. Wang, Numerical dissipation control in an adaptive wcns with a new smoothness indicator, Applied Mathematics and Computation 330 (2018) 239 - 253. doi:10.1016/j. amc.2018.01.019.

[46] O. San, K. Kara, Evaluation of riemann flux solvers for weno reconstruction schemes: Kelvin-helmholtz instability, Computers \& Fluids 117 (2015) 24-41. doi:10.1016/j.compfluid.2015.04.026 